\title{
24. ORGANIC CARBON ACCUMULATION AT SOUTHEAST GREENLAND SITE 918: IMPLICATIONS ON PALEOENVIRONMENT AND PALEOCEANOGRAPHY DURING LATE CENOZOIC TIMES ${ }^{1}$
}

\author{
Rainer $\operatorname{Stax}^{2}$
}

\begin{abstract}
Site 918 was drilled in the western part of the Irminger Basin to sample sediments comprising major changes in paleoclimate and paleoceanography of the last $51 \mathrm{~m}$.y. Organic geochemical investigations were performed to study the quality and quantity of organic matter within the distinct lithologic units. At Site 918, changes in the lithologic composition recognized in the sedimentary column correlate very well with changes in the organic fraction, which is used for paleoenvironmental reconstructions. The accumulation of organic carbon at Site 918 is mainly controlled by the interaction between surface-water productivity, bottom-water oxygenation, burial rates, and terrigenous organic supply. One of the main organic constituents is plant detritus deposited in preglacial times. Planktonic organic material dominates during glacial times (i.e., the last 5 m.y.).
\end{abstract}

\section{INTRODUCTION}

The Southeast Greenland Transect, Ocean Drilling Program (ODP) Leg 152, was drilled from September to November 1993 to gain information about (1) the nature and subsidence history of the seaward-dipping reflector sequence (SDRS), (2) the paleoceanographic and glaciation history of the southeast Greenland shelf and the Irminger Basin, and (3) the evolution of the break-up volcanism of the North Atlantic (Larsen, Saunders, Clift, et al., 1994). The sediments of the Irminger Basin probably record several important paleoceanographic events (e.g., the initial overflow of the North Atlantic Deep Water [NADW] and the onset of major Northern Hemisphere glaciations).

Site 918 is located on the upper continental rise, approximately $130 \mathrm{~km}$ off the east Greenland coast, at a water depth of $1868.5 \mathrm{~m}$ (Fig. 1; Larsen, Saunders, Clift, et al., 1994). The volcanic basement of the SDRS is overlain by about $1200 \mathrm{~m}$ of sediment, spanning in age from Paleocene to Holocene. As already shown by shipboard analyses, total organic carbon (TOC) contents of the sediments recovered at Site 918 are relatively low, ranging between 0.1 and 0.5 $\mathrm{wt} \%$ (Fig. 2). In this study, the major aim is to investigate the quantity and quality of organic matter in terms of a correlation of changes in the composition with changes in lithology and, thus, in paleoclimatic and paleoceanographic conditions. Several different methods were applied to study the organic carbon fraction of sediments at Site 918: elemental analyses (total organic carbon and total nitrogen content), Rock-Eval pyrolysis parameters, maceral microscopy, and specific biomarker extraction.

\section{Major Lithologies at Site 918}

The sedimentary sequence of Site 918 comprises six major lithologic units (cf. Larsen, Saunders, Clift, et al., 1994). Unit I (0-600 m below seafloor [mbsf]; Quaternary to late Miocene age) contains five subunits and mainly consists of dark gray silt with volcanic and nonvolcanic components. Ice-rafted debris (IRD) and dropstones occur throughout the entire unit except in Subunit IE. Lithologic Unit II (600-806.5 mbsf; early to late Miocene age) comprises nannofossil

${ }^{1}$ Saunders, A.D., Larsen, H.C., and Wise, S.W., Jr. (Eds.), 1998. Proc. ODP, Sci. Results, 152: College Station, TX (Ocean Drilling Program).

${ }^{2}$ Institute for Geology and Mineralogy, Erlangen University, Schloßgarten 5, 91054 Erlangen, Federal Republic of Germany. rstax@geol.uni-erlangen.de chalk and very dark gray silt with nannofossils. Sediments of this unit are heavily bioturbated and contain bivalve shells, sponge spicules, and radiolarians. In contrast to the carbonate-rich chalks of Unit II, the sediments of Unit III (806.5-1108.2 mbsf; late Oligocene age) are characterized by massive or laminated sand, silt, and frequently interbedded nannofossil chalks. Lithologic Unit IV (1108.2-1157.9 mbsf; early to middle Eocene age) consists of nannofossil chalk and silt with nannofossils, whereas Unit V (1157.9-1189.4 mbsf; early Eocene age) is dominated by glauconitic sands with interbedded calcareous sands. Remarkable is the lack of microfossils in Unit V (Larsen, Saunders, Clift, et al., 1994). The top of the basaltic pile is described in lithologic Unit VI (1189.4-1204.4 mbsf; unknown age).

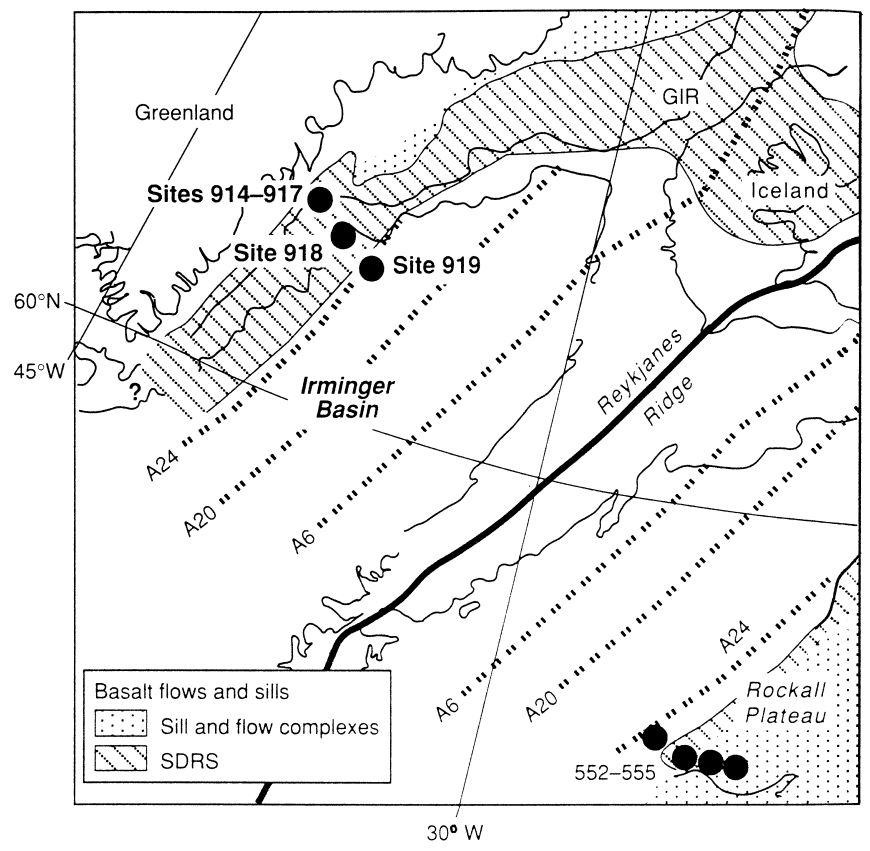

Figure 1. Map of the southeast Greenland Margin with locations of ODP Sites 914-919 and DSDP Sites 552-555. SDRS = seaward-dipping reflector sequence. GIR = Greenland-Iceland Ridge. A6-A24 indicate geomagnetic anomalies. 


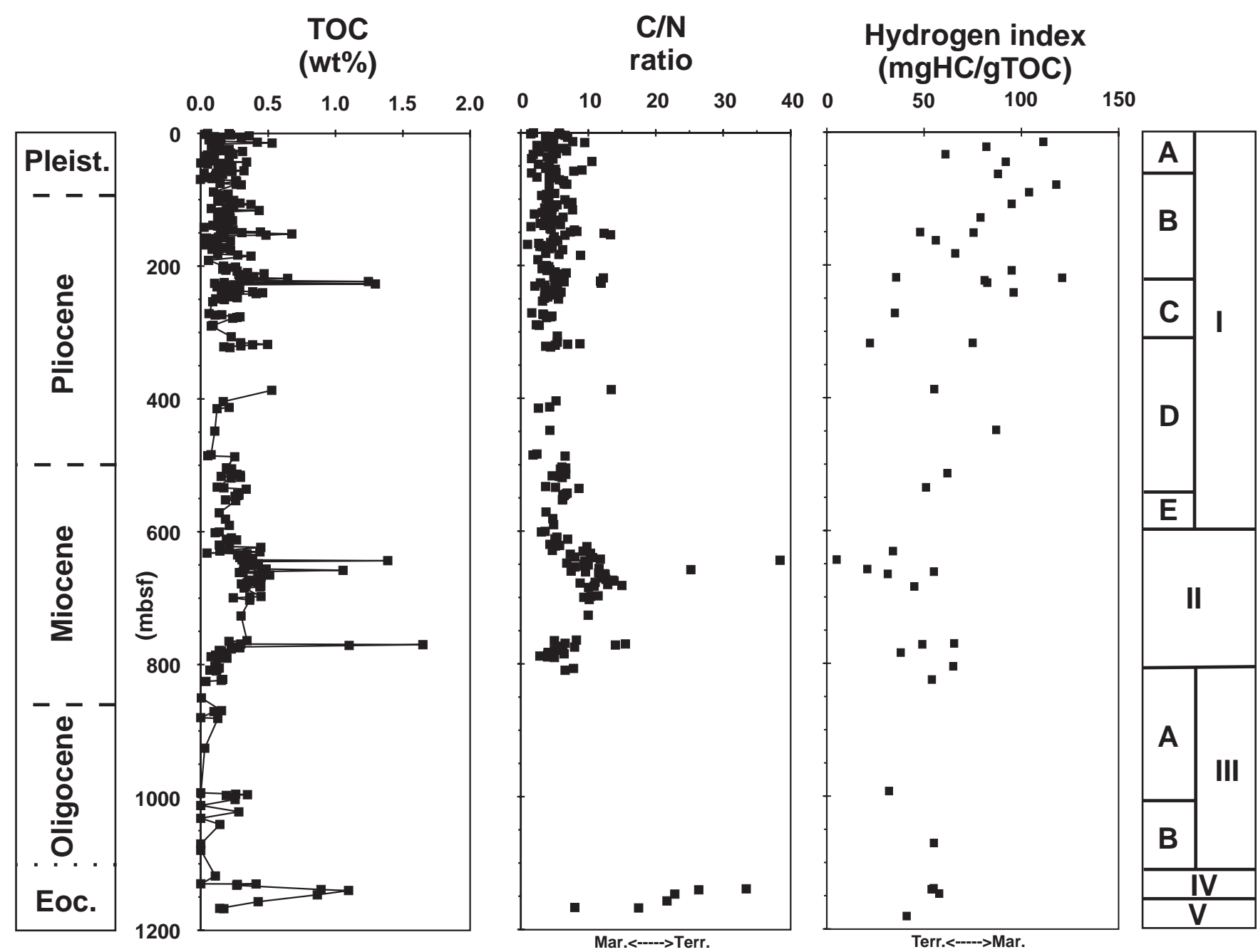

Figure 2. Total organic carbon, total organic carbon/total nitrogen ratios, and hydrogen index of Site 918 sediments vs. depth values. Left column indicates ages after preliminary shipboard stratigraphy (Larsen, Saunders, Clift, et al., 1994). Right column indicates lithologic units.

\section{METHODS}

Most of the TOC values are from shipboard data, measured following the analytical procedure described in the "Explanatory Notes" chapter in Larsen, Saunders, Clift, et al. (1994). Additional shorebased samples from Site 918 were examined for total carbon (TC), TOC, and total nitrogen using a Heraeus CHN Analyzer. To separate the organic carbon from carbonate-bonded carbon, the bulk samples were treated with $\mathrm{HCl}(10 \%)$, washed, and dried (Froelich, 1980; Weliky et al., 1983). The accuracy of the $\mathrm{CHN}$ analysis is $0.02 \%$, and the relative standard deviation of control measurements about $0.2 \%$. The carbonate content was calculated as

$$
\mathrm{CaCO}_{3}=(\mathrm{TC}-\mathrm{TOC}) \times 8.333,
$$

where TC and TOC are given in weight percent (wt\%) of the bulk sample. This calculation assumes that all carbonate in the sediment is calcite. The organic carbon/total nitrogen $(\mathrm{C} / \mathrm{N})$ ratio was calculated as an indicator for the composition of the organic carbon from TOC and total nitrogen content. Marine phyto- and zooplankton are characterized by mean $\mathrm{C} / \mathrm{N}$ ratios of $6-10$, whereas terrigenous organic matter shows values of more than 15 .

For an initial characterization of the organic material, Rock-Eval pyrolysis measurements were performed on ground bulk samples us- ing a Delsi, Inc., Rock-Eval II instrument and following the standard procedures described by Espitalié et al. (1977). The Rock-Eval parameters used are the hydrogen index (HI corresponds to the quantity of pyrolyzable hydrocarbons per gram TOC; $\mathrm{mg} \mathrm{HC} / \mathrm{g} \mathrm{C}$ ) and the oxygen index (OI corresponds to the quantity of carbon dioxide per gram TOC; $\mathrm{mg} \mathrm{CO}_{2} / \mathrm{g} \mathrm{C}$ ). In a van Krevelen diagram, a classification of the organic matter in terms of marine vs. terrigenous kerogen type is possible (see "Results" section below). In carbon-lean sediments, the organic matter may be strongly bonded to clay minerals (mineral matrix effect), which results in lower values. These values have to be interpreted cautiously (Peters, 1986; Katz, 1983). The temperature of maximum hydrocarbon yield during pyrolysis $\left(\mathrm{T}_{\max }\right)$ provides additional information about the maturity of the organic matter in the sediment. Immature organic matter has $\mathrm{T}_{\max }$ values of less than $435^{\circ} \mathrm{C}$ (Tissot and Welte, 1984).

Long-chain $n$-alkanes are very indicative biomarkers for the composition of organic material. $N$-alkanes from $\mathrm{C}_{27}$ through $\mathrm{C}_{31}$ point to a terrigenous source, whereas $n$-alkanes with lower molecular weights $\left(\mathrm{C}_{15}\right.$ through $\left.\mathrm{C}_{19}\right)$ mainly derive from marine organisms.

To examine the ground bulk sample for $n$-alkanes, the sediments were treated successively with methanol, methanol/dichloromethane (1:1), and dichloromethane (cf. Farrimond et al., 1990). After centrifuging, the three extracts were combined, and squalene was added as an internal standard. The hydrocarbon fraction was removed from the bulk extract by column chromatography, eluted with hexane, and in- 
jected into a HP (Series II) gas chromatograph. Helium was used as the carrier gas. An HP1 capillary column was used $(50 \mathrm{~m} \times 0.32 \mathrm{~mm}$; $0.17-\mu \mathrm{m}$ film of dimethyl-polysiloxane), and the temperature program was as follows: $60^{\circ} \mathrm{C}$ for $1 \mathrm{~min} ; 60^{\circ}-150^{\circ} \mathrm{C}$ at $10^{\circ} \mathrm{C} / \mathrm{min} ; 150^{\circ}-$ $300^{\circ} \mathrm{C}$ at $4^{\circ} \mathrm{C} / \mathrm{min}$, and isothermal at $300^{\circ} \mathrm{C}$ for $45 \mathrm{~min}$.

Microscopic investigation of the maceral composition was performed on a selected set of samples distributed over the entire core. Due to the low organic carbon content and the extremely small particle size, the amounts of macerals were estimated and presented as trends. To distinguish between marine and terrigenous organic particles, the maceral classification was performed according to Stach et al. (1982) and Hutton et al. (1980). Vitrinites, pollenites (terrigenous), and alginites (marine) are the dominant components; however, if these macerals are strongly destroyed, the terms lipto- and vitrodetrinite were used to be indicative of the presence of marine and terrigenous organic matter, respectively.

\section{RESULTS Quantity of Organic Carbon}

The sediments drilled at Site 918 are characterized by low to very low organic carbon contents throughout the cores (Fig. 2; Table 1). Most of the values are between 0 and $0.5 \mathrm{wt} \%$, which is within the fluctuation observed in sediments of the North Atlantic Ocean (e.g., Eldholm, Thiede, Taylor, et al., 1987; Srivastava, Arthur, Clement, et al., 1987). In a few samples, higher values of $1-1.5 \mathrm{wt} \%$ TOC were measured.

The lowermost part of the sediment column at Site 918 (Eocene glauconitic sands of lithologic Units V and IV) shows high organic carbon contents, whereas in the Oligocene interval the values decrease (up to $0.4 \mathrm{wt} \%$ ). A distinct increase in TOC can be recognized in the Miocene sediments (500-800 mbsf), with a maximum at about $650 \mathrm{mbsf}$ (Fig. 2). This interval also includes some single spikes, with values as high as $1.6 \mathrm{wt} \%$. The Pliocene-Pleistocene sediments are characterized by a higher fluctuation of the TOC contents, with values between 0 and $0.5 \mathrm{wt} \%$.

\section{Composition of Organic Carbon}

For paleoenvironmental interpretations, the information about marine and terrigenous proportions of the organic carbon fraction is absolutely necessary. To determine the quality of the organic matter, several different methods were used. The interpretation of the data is based on the combination of the different approaches because all methods have their specific limits (cf. Stein, 1991). Data from RockEval pyrolysis, kerogen microscopy, biomarker ( $n$-alkane) extractions, and organic carbon/nitrogen ratios were used in this study to gain information about the composition and provenance of the organic matter.

\section{Rock-Eval Data}

Rock-Eval pyrolysis provides a rapid evaluation method of organic carbon. Due to the mineral matrix effect (Katz, 1983), however, the values are relatively low, and only trends are important for the distinction of marine vs. terrigenous origin. The sediments of Site 918 generally contain a mixture of marine and terrigenous organic matter. Based on the hydrogen index values, the record can be subdivided into a lower, terrigenous-influenced interval (1150-500 mbsf; 5-60 $\mathrm{mg} \mathrm{HC/g} \mathrm{TOC)} \mathrm{and} \mathrm{an} \mathrm{upper,} \mathrm{marine-dominated} \mathrm{section} \mathrm{(500-0}$ mbsf; 20-110 mg HC/g TOC; Fig. 2; Table 2).

\section{C/N Ratios}

Organic carbon/total nitrogen ratios were used to characterize the quality of the organic matter (Scheffer and Schachtschabel, 1984; Stein, 1991; Stax, 1994). The data vary between 3 and 15, with max- imum values up to 36 at 650 and 1150 mbsf (Fig. 2; Table 1). These spikes are probably the result of an enhanced content of wood pieces in the sediment. In most of the Eocene-Oligocene samples, the total nitrogen content is below the detection limit, indicating a terrigenous organic matter source. A slight maximum between 600 and $800 \mathrm{mbsf}$ coincides with high organic carbon values, indicating increased terrigenous organic matter accumulation during this time interval.

\section{Maceral Data}

Kerogen microscopy was performed on 11 resin-impregnated specimens; the results are shown in Table 3. The Eocene-Oligocene sediments are dominated by terrigenous macerals as vitrinite and vitrodetrinite. Pollenites are rare, and background fluorescence is very low. Abundant wood pieces show the characteristic tissue structure.

In the middle Miocene interval (lithologic Unit II) background fluorescence increases, whereas single macerals disappear. Vitrodetrinite and liptodetrinite dominate in the maceral composition. Pollenite is rare.

The Pliocene-Pleistocene interval displays a very low maceral content (Table 3). Background fluorescence intensity as an indicator for marine organic matter varies between medium and low. Terrigenous macerals are absent.

\section{Biomarker Data}

Biomarkers (e.g., $n$-alkanes) may give important information about the composition of organic matter [Prahl and Muehlhausen, 1989]). The aliphatic fraction of the lower part of Site 918 is dominated by long-chain $n$-alkanes $\left(\mathrm{C}_{27}\right.$ through $\left.\mathrm{C}_{31}\right)$, indicating a terrigenous source of organic matter (Fig. 3; Table 4). In the middle and upper part of the sedimentary sequence, the pattern points to a mixture of marine and terrigenous organic carbon. However, due to the very low organic carbon content the biomarker data have to be interpreted very cautiously and need confirmation by further analyses.

\section{Flux of Organic Carbon}

Mass accumulation (flux) rates were calculated for the upper 3.6 m.y. to interpret the data in terms of organic carbon supply (cf. van Andel et al., 1975). The values are based on shipboard stratigraphy and index property data (Larsen, Saunders, Clift, et al., 1994; Fig. 4; Table 1). Between 3.6 and $2.4 \mathrm{Ma}$, the flux rates of organic carbon are relatively high (up to $0.35 \mathrm{~g} / \mathrm{cm}^{2} / \mathrm{k}$.y.). Most of the values in this time interval vary between 0.01 and $0.2 \mathrm{~g} / \mathrm{cm}^{2} / \mathrm{k}$.y. During the last 2.4 m.y., they decrease below $0.05 \mathrm{~g} / \mathrm{cm}^{2} / \mathrm{k}$.y.

\section{DISCUSSION AND CONCLUSIONS}

The marine depositional environment of the Southeast Greenland Margin has undergone strong changes in climate, sea level, and surface-water circulation during the last $51 \mathrm{~m}$.y. These changes regard the lithogenic sediment fraction as well as the organic material in the sediment. For paleoenvironmental reconstructions using the sedimentary record, several different organic and inorganic geochemical, physical, and petrological methods can be applied. Quality and quantity of the organic sedimentary fraction are very important parameters to characterize a depositional system. Climate-induced changes in the siliciclastic fraction of the sediment necessarily lead to changes in the organic fraction; thus, a combined investigation can help to get insight into the paleoenvironmental conditions of the region. In the following, the results of a investigation of the organic matter are interpreted to reconstruct the temporal changes in climatic conditions of the Southeast Greenland Continental Margin. Interpretations are presented in the three main time slices, also recognized as lithologic units in the sedimentary column. 
Table 1. Summary table of Site 918 data.

\begin{tabular}{|c|c|c|c|c|c|c|c|c|c|c|}
\hline & & & & & & & & & & ccumulation ra \\
\hline $\begin{array}{l}\text { Core, section, } \\
\text { interval }(\mathrm{cm})\end{array}$ & $\begin{array}{l}\text { Depth } \\
\text { (mbsf) }\end{array}$ & $\begin{array}{l}\text { Age } \\
\text { (Ma) }\end{array}$ & $\begin{array}{c}\text { TC } \\
\text { (wt \%) }\end{array}$ & $\begin{array}{c}\text { IC } \\
\text { (wt\%) }\end{array}$ & $\begin{array}{l}\text { TOC } \\
\text { (wt \%) }\end{array}$ & $\begin{array}{l}\mathrm{CaCO}_{3} \\
(\mathrm{wt} \%)\end{array}$ & $\begin{array}{c}\mathrm{TN} \\
(\mathrm{wt} \%)\end{array}$ & $\begin{array}{c}\mathrm{TOC} / \mathrm{N} \\
\text { ratio }\end{array}$ & $\begin{array}{c}\text { Bulk } \\
\text { (g/cm²/k.y.) }\end{array}$ & $\begin{array}{c}\text { TOC } \\
\left(\mathrm{g} / \mathrm{cm}^{2} / \mathrm{k} . \mathrm{y} .\right)\end{array}$ \\
\hline 152-918A- & & & & & & & & & & \\
\hline $1 \mathrm{H}-1,33-34$ & 0.33 & 0.006 & 0.52 & 0.47 & 0.05 & 3.92 & 0.03 & 2 & 8.400 & 0.004 \\
\hline $1 \mathrm{H}-1,62-63$ & 0.62 & 0.010 & 0.51 & 0.30 & 0.21 & 2.50 & 0.04 & 6 & 6.720 & 0.014 \\
\hline $1 \mathrm{H}-2,28-29$ & 1.78 & 0.030 & 0.27 & 0.24 & 0.03 & 2.00 & 0.02 & 1 & 9.920 & 0.003 \\
\hline $2 \mathrm{H}-1,73-74$ & 2.53 & 0.042 & 0.55 & 0.33 & 0.22 & 2.75 & 0.04 & 6 & 9.920 & 0.022 \\
\hline $2 \mathrm{H}-2,58-59$ & 3.88 & 0.065 & 0.54 & 0.18 & 0.36 & 1.50 & 0.06 & 7 & 7.600 & 0.027 \\
\hline $2 \mathrm{H}-3,33-34$ & 5.13 & 0.086 & 0.21 & 0.11 & 0.10 & 0.92 & 0.03 & 4 & 9.600 & 0.010 \\
\hline $2 \mathrm{H}-4,65-66$ & 6.95 & 0.116 & 0.42 & 0.12 & 0.30 & 1.00 & 0.04 & 7 & 8.320 & 0.025 \\
\hline $2 \mathrm{H}-5,21-22$ & 8.01 & 0.134 & 0.29 & 0.14 & 0.15 & 1.17 & 0.03 & 4 & $\begin{array}{l}0.240 \\
10.240\end{array}$ & 0.015 \\
\hline $2 \mathrm{H}-6,51-52$ & $\begin{array}{l}0.01 \\
9.81\end{array}$ & 0.164 & 0.19 & 0.13 & 0.06 & 1.08 & 0.01 & $\begin{array}{l}4 \\
4\end{array}$ & 12.800 & 0.008 \\
\hline $3 \mathrm{H}-1,36-37$ & 11.66 & 0.194 & 0.18 & 0.09 & 0.09 & 0.75 & 0.02 & 5 & 12.240 & 0.011 \\
\hline $3 \mathrm{H}-2,73-74$ & 13.53 & 0.226 & 0.57 & 0.15 & 0.42 & 1.25 & 0.05 & 8 & 9.360 & 0.039 \\
\hline $3 \mathrm{H}-3,30-31$ & 14.60 & 0.243 & 0.69 & 0.16 & 0.53 & 1.33 & 0.06 & 9 & 8.880 & 0.047 \\
\hline $3 \mathrm{H}-4,27-28$ & 16.07 & 0.268 & 0.37 & 0.27 & 0.10 & 2.25 & 0.03 & 4 & 12.000 & 0.012 \\
\hline $3 \mathrm{H}-6,27-28$ & 19.07 & 0.318 & 0.56 & 0.48 & 0.08 & 4.00 & 0.03 & 2 & 10.240 & 0.008 \\
\hline $3 \mathrm{H}-7,38-39$ & 20.68 & 0.345 & 0.27 & $\begin{array}{l}0.40 \\
0.13\end{array}$ & 0.14 & 1.08 & 0.04 & 4 & 11.360 & 0.016 \\
\hline $4 \mathrm{H}-1,76-77$ & 21.56 & 0.359 & 0.28 & 0.16 & 0.12 & 1.33 & 0.03 & 4 & 11.600 & 0.014 \\
\hline $4 \mathrm{H}-2,125-126$ & 23.55 & 0.393 & 0.33 & 0.21 & 0.12 & 1.75 & 0.02 & 6 & 12.160 & 0.015 \\
\hline $4 \mathrm{H}-3,32-33$ & 24.12 & 0.402 & 0.31 & 0.10 & 0.21 & 0.83 & 0.04 & 5 & 10.400 & 0.022 \\
\hline $4 \mathrm{H}-4,36-37$ & 25.66 & 0.428 & 0.25 & 0.08 & 0.17 & 0.67 & 0.04 & 5 & 12.800 & 0.022 \\
\hline $4 \mathrm{H}-5,63-64$ & 27.43 & 0.457 & 0.45 & 0.14 & 0.31 & 1.17 & 0.05 & 7 & 10.480 & 0.032 \\
\hline $4 \mathrm{H}-6,31-32$ & 28.61 & 0.477 & 1.06 & 0.96 & 0.10 & 8.00 & 0.04 & 3 & 7.760 & 0.008 \\
\hline $4 \mathrm{H}-7,13-14$ & 29.93 & 0.499 & 0.29 & 0.07 & 0.22 & 0.58 & 0.05 & 5 & 9.520 & 0.021 \\
\hline $5 \mathrm{H}-1,126-127$ & 31.56 & 0.526 & 0.46 & 0.22 & 0.24 & 1.83 & 0.05 & 5 & 9.920 & 0.024 \\
\hline $5 \mathrm{H}-2,80-81$ & 32.60 & 0.543 & 0.13 & 0.08 & 0.05 & 0.67 & 0.03 & 2 & 11.840 & 0.006 \\
\hline $5 \mathrm{H}-3,128-129$ & 34.58 & 0.576 & 0.10 & 0.06 & 0.04 & 0.50 & 0.00 & & 13.680 & 0.005 \\
\hline $5 \mathrm{H}-4,126-127$ & 36.06 & 0.601 & 0.18 & 0.08 & 0.10 & 0.67 & 0.03 & 4 & 13.040 & 0.013 \\
\hline $5 \mathrm{H}-5,133-134$ & 37.63 & 0.627 & $\begin{array}{l}0.10 \\
0.49\end{array}$ & 0.43 & 0.06 & 3.58 & 0.00 & 4 & 11.280 & 0.007 \\
\hline $5 \mathrm{H}-6,132-133$ & 39.12 & 0.652 & 0.12 & 0.04 & 0.08 & 0.33 & 0.05 & 2 & 10.000 & 0.008 \\
\hline $5 \mathrm{H}-7,27-28$ & 39.57 & 0.660 & 0.27 & 0.06 & 0.21 & 0.50 & 0.04 & 5 & 11.520 & 0.024 \\
\hline $6 \mathrm{H}-2,40-41$ & 41.70 & 0.695 & 0.38 & 0.31 & 0.07 & 2.58 & 0.00 & & 7.200 & 0.005 \\
\hline $6 \mathrm{H}-3,41-42$ & 43.21 & 0.720 & 0.80 & 0.46 & 0.34 & 3.83 & 0.03 & 11 & 8.720 & 0.030 \\
\hline $6 \mathrm{H}-4,40-41$ & 44.70 & 0.745 & 0.20 & 0.21 & 0.00 & 1.75 & 0.00 & & 13.200 & 0.000 \\
\hline $6 \mathrm{H}-5,40-41$ & 46.20 & 0.770 & 0.26 & 0.21 & 0.05 & 1.75 & 0.00 & & 12.480 & 0.006 \\
\hline $6 \mathrm{H}-6,33-34$ & 47.63 & 0.794 & 0.74 & 0.61 & 0.13 & 5.08 & 0.05 & 3 & 9.520 & 0.012 \\
\hline $6 \mathrm{H}-7,41-42$ & 49.21 & 0.820 & 0.31 & 0.08 & 0.23 & 0.67 & 0.05 & 4 & 11.120 & 0.026 \\
\hline $7 \mathrm{H}-1,122-123$ & 50.52 & 0.842 & 0.95 & 0.79 & 0.16 & 6.58 & 0.00 & 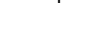 & 9.920 & 0.016 \\
\hline $7 \mathrm{H}-2,30-31$ & 51.10 & 0.852 & 0.40 & 0.24 & 0.16 & 2.00 & 0.04 & 4 & 10.720 & 0.017 \\
\hline $7 \mathrm{H}-3,123-124$ & 53.53 & 0.892 & 0.25 & 0.12 & 0.13 & 1.00 & 0.04 & 4 & 11.040 & 0.014 \\
\hline $7 \mathrm{H}-4,112-113$ & 54.92 & 0.915 & 0.30 & 0.09 & 0.21 & 0.75 & 0.04 & 5 & 12.160 & 0.026 \\
\hline $7 \mathrm{H}-5,111-112$ & 56.41 & 0.940 & 0.42 & 0.10 & 0.32 & 0.83 & 0.04 & 9 & 11.600 & 0.037 \\
\hline $7 \mathrm{H}-6,94-95$ & 57.74 & 0.962 & 0.38 & 0.15 & 0.23 & 1.25 & 0.03 & 8 & 11.520 & 0.027 \\
\hline $8 \mathrm{H}-1,139-140$ & 60.19 & 1.803 & 0.46 & 0.34 & 0.12 & 2.83 & 0.02 & 5 & 10.650 & 0.012 \\
\hline $8 \mathrm{H}-2,34-35$ & 60.64 & 1.810 & 0.09 & 0.07 & 0.02 & 0.58 & 0.01 & 2 & 12.225 & 0.003 \\
\hline $8 \mathrm{H}-3,34-35$ & 62.14 & 1.832 & 0.33 & 0.17 & 0.16 & 1.42 & 0.03 & 5 & 9.750 & 0.016 \\
\hline $8 \mathrm{H}-4,23-24$ & 63.53 & 1.853 & 0.51 & 0.40 & 0.11 & 3.33 & 0.03 & 4 & 10.500 & 0.012 \\
\hline $8 \mathrm{H}-5,125-126$ & 66.05 & 1.891 & 0.14 & 0.04 & 0.10 & 0.33 & 0.02 & 5 & 12.000 & 0.012 \\
\hline $8 \mathrm{H}-6,35-36$ & 66.65 & 1.900 & 0.13 & 0.07 & 0.06 & 0.58 & 0.03 & 2 & 11.775 & 0.007 \\
\hline $8 \mathrm{H}-7,33-34$ & 68.13 & 1.922 & 0.15 & 0.06 & 0.09 & 0.50 & 0.02 & 5 & 12.150 & 0.010 \\
\hline $9 \mathrm{H}-1,127-128$ & 69.57 & 1.944 & 0.95 & 0.95 & 0.00 & 7.91 & 0.03 & & 8.775 & 0.000 \\
\hline $9 \mathrm{H}-2,113-114$ & 70.93 & 1.964 & 0.39 & 0.13 & 0.26 & 1.08 & 0.05 & 6 & 8.175 & 0.021 \\
\hline $9 \mathrm{H}-3,114-115$ & 72.44 & 1.987 & 0.33 & 0.06 & 0.27 & 0.50 & 0.04 & 6 & 10.050 & 0.027 \\
\hline $9 \mathrm{H}-5,113-114$ & 75.43 & 2.031 & 0.16 & 0.02 & 0.14 & 0.17 & 0.03 & 4 & 10.050 & 0.014 \\
\hline $9 \mathrm{H}-7,37-38$ & 77.67 & 2.065 & 0.36 & 0.06 & 0.30 & 0.50 & 0.04 & 7 & 10.650 & 0.032 \\
\hline $9 \mathrm{H}-6,112-113$ & 76.92 & 2.054 & 0.34 & 0.08 & 0.26 & 0.67 & 0.04 & 6 & 10.275 & 0.027 \\
\hline $11 \mathrm{H}-1,122-123$ & 88.52 & 2.228 & 0.34 & 0.25 & 0.09 & 2.08 & 0.02 & 4 & 10.875 & 0.010 \\
\hline $11 \mathrm{H}-3,110-111$ & 91.40 & 2.271 & 0.24 & 0.04 & 0.20 & 0.33 & 0.04 & 5 & 10.350 & 0.021 \\
\hline $11 \mathrm{H}-4,104-105$ & 92.84 & 2.293 & 0.21 & 0.05 & 0.16 & 0.42 & 0.04 & 4 & 8.025 & 0.013 \\
\hline $11 \mathrm{H}-5,105-106$ & 94.35 & 2.315 & 0.17 & 0.04 & 0.13 & 0.33 & 0.04 & 3 & 6.900 & 0.009 \\
\hline $12 \mathrm{H}-1,109-110$ & 97.89 & 2.368 & 0.36 & 0.17 & 0.19 & 1.42 & 0.05 & 4 & 9.675 & 0.018 \\
\hline $12 \mathrm{H}-2,113-114$ & 99.43 & 2.391 & 0.18 & 0.05 & 0.13 & 0.42 & 0.03 & 4 & 12.375 & 0.016 \\
\hline $12 \mathrm{H}-3,113-114$ & 100.93 & 2.405 & 0.32 & 0.08 & 0.24 & 0.67 & 0.04 & 7 & 25.870 & 0.063 \\
\hline $12 \mathrm{H}-4,108-109$ & 102.38 & 2.413 & 0.18 & 0.05 & 0.13 & 0.42 & 0.03 & 4 & 27.860 & 0.037 \\
\hline $12 \mathrm{H}-6,70-71$ & 105.00 & 2.428 & 0.37 & 0.08 & 0.29 & 0.67 & 0.04 & 8 & 26.865 & 0.078 \\
\hline $13 \mathrm{H}-1,101-102$ & 107.31 & 2.440 & 0.46 & $\begin{array}{l}0.09 \\
0.09\end{array}$ & 0.37 & 0.75 & 0.05 & $\begin{array}{l}0 \\
7\end{array}$ & 26.467 & 0.098 \\
\hline $13 \mathrm{H}-2,99-100$ & 108.79 & 2.448 & 0.34 & 0.10 & 0.24 & 0.83 & 0.05 & 5 & 26.268 & 0.064 \\
\hline $13 \mathrm{H}-3,99-100$ & 110.29 & 2.457 & 0.33 & 0.11 & 0.22 & 0.92 & 0.05 & 4 & 25.671 & 0.055 \\
\hline $13 \mathrm{H}-4,97-98$ & 111.77 & 2.465 & 0.27 & 0.08 & 0.19 & 0.67 & 0.04 & 5 & 26.865 & 0.051 \\
\hline $13 \mathrm{H}-5,118-119$ & 113.48 & 2.474 & 0.18 & 0.10 & 0.08 & 0.83 & 0.02 & 4 & 27.661 & 0.021 \\
\hline $13 \mathrm{H}-6,117-118$ & 114.97 & 2.482 & 0.40 & 0.19 & 0.21 & 1.58 & 0.05 & 5 & 28.059 & 0.060 \\
\hline $14 \mathrm{H}-1,34-35$ & 116.14 & 2.489 & 0.63 & 0.20 & 0.43 & $\begin{array}{l}1.60 \\
1.67\end{array}$ & 0.06 & 8 & 26.865 & 0.116 \\
\hline $14 \mathrm{H}-2,76-77$ & 118.06 & 2.499 & 0.34 & 0.13 & 0.21 & 1.08 & 0.05 & 4 & 28.059 & 0.058 \\
\hline $14 \mathrm{H}-3,121-122$ & 120.01 & 2.510 & 0.25 & 0.11 & 0.14 & 0.92 & 0.04 & 3 & 27.860 & 0.039 \\
\hline $14 \mathrm{H}-4,87-88$ & 121.17 & 2.516 & 0.22 & 0.07 & 0.15 & 0.58 & 0.04 & 4 & 26.268 & 0.040 \\
\hline $14 \mathrm{H}-5,56-57$ & 122.36 & 2.523 & 0.27 & 0.10 & 0.17 & 0.83 & 0.08 & 2 & 27.064 & 0.046 \\
\hline $15 \mathrm{H}-1,121-122$ & 126.51 & 2.546 & 0.22 & 0.09 & 0.13 & 0.75 & 0.03 & 5 & 29.054 & 0.037 \\
\hline $15 \mathrm{H}-2,31-32$ & 127.11 & 2.549 & 0.37 & 0.15 & 0.22 & 1.25 & 0.03 & 6 & 29.054 & 0.063 \\
\hline $15 \mathrm{H}-3,120-121$ & 129.50 & 2.562 & 0.38 & 0.21 & 0.17 & 1.75 & 0.04 & 4 & 28.855 & 0.049 \\
\hline $15 \mathrm{H}-4,120-121$ & 131.00 & 2.571 & 0.37 & 0.21 & 0.16 & 1.75 & 0.04 & 4 & 30.049 & 0.047 \\
\hline $15 \mathrm{H}-5,31-32$ & 131.61 & 2.574 & 0.36 & 0.13 & 0.23 & 1.08 & 0.04 & 5 & 31.044 & 0.073 \\
\hline $16 \mathrm{H}-1,33-34$ & 133.63 & 2.585 & 0.26 & 0.09 & 0.17 & 0.75 & 0.04 & 5 & 26.666 & 0.046 \\
\hline $16 \mathrm{H}-2,78-79$ & 135.58 & 2.596 & 0.29 & 0.09 & 0.20 & 0.75 & 0.05 & 4 & 27.860 & 0.057 \\
\hline $16 \mathrm{H}-3,29-30$ & 136.59 & 2.601 & 0.21 & 0.07 & 0.14 & 0.58 & 0.05 & 3 & 29.452 & 0.041 \\
\hline $16 \mathrm{H}-4,73-74$ & 138.53 & 2.612 & 0.18 & 0.09 & 0.09 & 0.75 & 0.02 & 6 & 32.636 & 0.029 \\
\hline $16 \mathrm{H}-5,30-31$ & 139.60 & 2.618 & 0.21 & 0.09 & 0.12 & 0.75 & 0.04 & 3 & 28.656 & 0.036 \\
\hline $16 \mathrm{H}-6,78-79$ & 141.58 & 2.629 & 0.16 & 0.14 & 0.02 & 1.17 & 0.02 & 1 & 37.810 & 0.009 \\
\hline
\end{tabular}


Table 1 (continued).

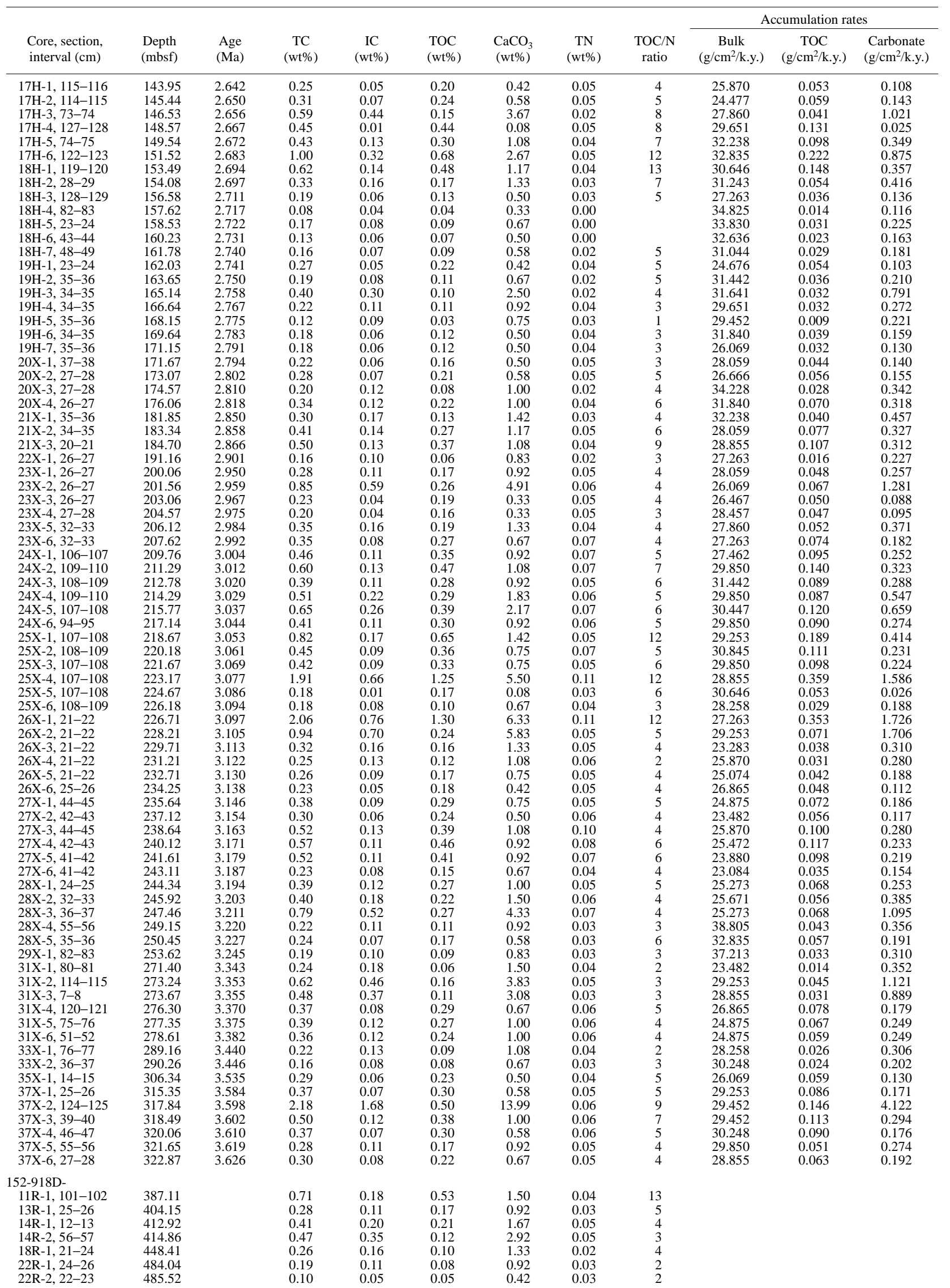


Table 1 (continued).

\begin{tabular}{|c|c|c|c|c|c|c|c|c|}
\hline & & & & & & & & \\
\hline $\begin{array}{l}\text { Core, section, } \\
\text { interval }(\mathrm{cm})\end{array}$ & $\begin{array}{l}\text { Depth } \\
\text { (mbsf) }\end{array}$ & $\begin{array}{l}\text { Age } \\
\text { (Ma) }\end{array}$ & $\begin{array}{c}\mathrm{TC} \\
(\mathrm{wt} \%)\end{array}$ & $\begin{array}{c}\mathrm{IC} \\
\text { (wt } \%)\end{array}$ & $\begin{array}{l}\text { TOC } \\
\text { (wt } \%)\end{array}$ & $\begin{array}{l}\mathrm{CaCO}_{3} \\
\text { (wt\%) }\end{array}$ & $\begin{array}{c}\mathrm{TN} \\
(\mathrm{wt} \%)\end{array}$ & $\begin{array}{c}\mathrm{TOC} / \mathrm{N} \\
\text { ratio }\end{array}$ \\
\hline $22 \mathrm{R}-3,33-35$ & 487.13 & & 0.31 & 0.06 & 0.25 & 0.50 & 0.04 & 7 \\
\hline $24 \mathrm{R}-1,40-41$ & 503.60 & & 0.91 & 0.72 & 0.19 & 6.00 & 0.03 & 6 \\
\hline $24 \mathrm{R}-2,39-40$ & 505.09 & & 0.49 & 0.26 & 0.23 & 2.17 & 0.03 & 7 \\
\hline $24 \mathrm{R}-3,47-48$ & 506.67 & & 0.56 & 0.35 & 0.21 & 2.92 & 0.04 & 6 \\
\hline $25 \mathrm{R}-1,40-42$ & 513.20 & & 0.55 & 0.28 & 0.27 & 2.33 & 0.04 & 6 \\
\hline $25 \mathrm{R}-2,50-51$ & 514.80 & & 0.55 & 0.26 & 0.29 & 2.17 & 0.04 & 7 \\
\hline $25 \mathrm{R}-3,100-102$ & 516.80 & & 0.23 & 0.08 & 0.15 & 0.67 & 0.03 & 5 \\
\hline $25 \mathrm{R}-4,58-60$ & 517.88 & & 4.17 & 3.88 & 0.29 & 32.32 & 0.05 & 6 \\
\hline $25 \mathrm{R}-5,49-50$ & 519.29 & & 0.45 & 0.23 & 0.22 & 1.92 & 0.04 & 6 \\
\hline $27 \mathrm{R}-1,81-82$ & 532.91 & & 0.18 & 0.06 & 0.12 & 0.50 & 0.03 & 4 \\
\hline $27 \mathrm{R}-2,37-38$ & 533.97 & & 0.24 & 0.07 & 0.17 & 0.58 & 0.03 & 5 \\
\hline $27 \mathrm{R}-3,71-72$ & 535.81 & & 0.40 & 0.06 & 0.34 & 0.50 & 0.04 & 9 \\
\hline 28R-1, 97-98 & 542.77 & & 0.34 & 0.07 & 0.27 & 0.58 & 0.04 & 7 \\
\hline 28R-2, 112-113 & 544.42 & & 0.34 & 0.06 & 0.28 & 0.50 & 0.04 & 7 \\
\hline $28 \mathrm{R}-3,73-74$ & 545.53 & & 0.39 & 0.12 & 0.27 & 1.00 & 0.04 & 6 \\
\hline $28 \mathrm{R}-4,42-43$ & 546.72 & & 0.31 & $\begin{array}{l}0.12 \\
0.06\end{array}$ & 0.25 & $\begin{array}{l}1.00 \\
0.50\end{array}$ & $\begin{array}{l}0.04 \\
0.04\end{array}$ & $\begin{array}{l}0 \\
6\end{array}$ \\
\hline $29 \mathrm{R}-1,56-57$ & 551.96 & & 0.25 & 0.07 & 0.18 & 0.58 & 0.03 & 6 \\
\hline $29 \mathrm{R}-2,29-30$ & 553.19 & & 0.30 & 0.04 & 0.26 & 0.33 & 0.04 & 6 \\
\hline $31 \mathrm{R}-1,77-78$ & 571.47 & & 0.40 & 0.26 & 0.14 & 2.17 & 0.04 & 4 \\
\hline 32R-1, 90-92 & 581.20 & & 0.31 & 0.13 & 0.18 & 1.08 & 0.04 & 5 \\
\hline $33 \mathrm{R}-1,32-33$ & 590.32 & & 0.58 & 0.37 & $\begin{array}{l}0.10 \\
0.21\end{array}$ & $\begin{array}{l}1.00 \\
3.08\end{array}$ & 0.04 & 5 \\
\hline $34 \mathrm{R}-1,90-91$ & 600.50 & & 0.27 & 0.13 & $\begin{array}{l}0.21 \\
0.14\end{array}$ & $\begin{array}{l}3.08 \\
1.08\end{array}$ & $\begin{array}{l}0.04 \\
0.04\end{array}$ & 4 \\
\hline $34 \mathrm{R}-2,43-45$ & 601.53 & & 0.37 & 0.26 & 0.11 & 2.17 & 0.04 & $\begin{array}{r}4 \\
3\end{array}$ \\
\hline $35 \mathrm{R}-1,14-15$ & 609.34 & & 0.43 & 0.20 & 0.23 & 1.67 & 0.04 & 5 \\
\hline $35 \mathrm{R}-2,73-74$ & 611.43 & & 0.30 & 0.11 & 0.19 & 0.92 & 0.04 & 5 \\
\hline 35R-CC, $3-4$ & 612.23 & & 0.48 & 0.22 & 0.26 & 1.83 & 0.04 & 7 \\
\hline $36 \mathrm{R}-1,117-118$ & 620.07 & & 0.99 & 0.85 & 0.14 & 7.08 & 0.03 & 4 \\
\hline 36R-2, 119-120 & $\begin{array}{l}020.01 \\
621.59\end{array}$ & & 0.87 & $\begin{array}{l}0.83 \\
0.66\end{array}$ & $\begin{array}{l}0.14 \\
0.21\end{array}$ & $\begin{array}{l}1.08 \\
5.50\end{array}$ & 0.04 & $\begin{array}{l}4 \\
6\end{array}$ \\
\hline $36 \mathrm{R}-3,53-54$ & 622.43 & & $\begin{array}{l}0.01 \\
1.41\end{array}$ & $\begin{array}{l}0.00 \\
1.20\end{array}$ & 0.21 & 10.00 & 0.04 & $\begin{array}{l}0 \\
5\end{array}$ \\
\hline $36 \mathrm{R}-\mathrm{CC}, 5-6$ & 623.45 & & $\begin{array}{l}1.41 \\
1.45\end{array}$ & 1.00 & 0.45 & 8.33 & 0.05 & 10 \\
\hline $37 \mathrm{R}-1,37-38$ & 628.97 & & 1.00 & 0.86 & 0.14 & 7.16 & 0.03 & 5 \\
\hline 37R-2, 18-19 & 630.28 & & 1.91 & 1.47 & 0.44 & 12.25 & 0.05 & 9 \\
\hline $37 \mathrm{R}-3,37-38$ & 631.97 & & $\begin{array}{l}4.36 \\
4.36\end{array}$ & $\begin{array}{l}1.41 \\
4.31\end{array}$ & 0.05 & 35.90 & 0.00 & \\
\hline $37 \mathrm{R}-4,25-26$ & $\begin{array}{l}031.911 \\
633.35\end{array}$ & & $\begin{array}{l}4.00 \\
1.02\end{array}$ & $\begin{array}{l}4.31 \\
0.68\end{array}$ & 0.34 & $\begin{array}{r}53.90 \\
5.66\end{array}$ & 0.03 & 10 \\
\hline $37 \mathrm{R}-5,26-27$ & 634.86 & & 3.28 & $\begin{array}{l}3.00 \\
3.01\end{array}$ & 0.27 & 25.07 & 0.04 & 7 \\
\hline $38 \mathrm{R}-1,88-90$ & 639.08 & & 2.87 & 2.58 & 0.29 & 21.49 & 0.04 & 8 \\
\hline $38 \mathrm{R}-2,25-26$ & 639.95 & & 1.05 & 0.67 & 0.38 & 5.58 & 0.04 & 11 \\
\hline $38 \mathrm{R}-3,118-120$ & 642.38 & & 0.84 & 0.53 & 0.31 & 4.41 & 0.03 & 12 \\
\hline $38 \mathrm{R}-4,105-106$ & 643.75 & & $\begin{array}{l}.04 \\
1.49\end{array}$ & 0.10 & 1.39 & 0.83 & 0.04 & 38 \\
\hline $38 \mathrm{R}-5,76-78$ & $\begin{array}{l}043.13 \\
644.96\end{array}$ & & $\begin{array}{l}1.49 \\
1.65\end{array}$ & $\begin{array}{l}0.10 \\
1.31\end{array}$ & $\begin{array}{l}1.39 \\
0.34\end{array}$ & $\begin{array}{r}0.83 \\
10.91\end{array}$ & $\begin{array}{l}0.04 \\
0.04\end{array}$ & $\begin{array}{r}58 \\
9\end{array}$ \\
\hline $39 \mathrm{R}-1,25-26$ & $\begin{array}{l}048.15 \\
648\end{array}$ & & 1.64 & 1.21 & 0.43 & $\begin{array}{l}10.91 \\
10.08\end{array}$ & 0.06 & 7 \\
\hline $39 \mathrm{R}-2,31-32$ & 649.71 & & $\begin{array}{l}2.04 \\
2.62\end{array}$ & $\begin{array}{l}1.21 \\
2.26\end{array}$ & 0.36 & $\begin{array}{l}10.00 \\
18.83\end{array}$ & 0.04 & 9 \\
\hline $39 \mathrm{R}-3,18-19$ & 651.08 & & 2.48 & 2.10 & 0.38 & 17.49 & 0.04 & 10 \\
\hline $39 \mathrm{R}-4,20-22$ & 652.60 & & 1.53 & 1.20 & 0.33 & 10.00 & 0.03 & 9 \\
\hline $39 \mathrm{R}-5,35-37$ & 654.25 & & 4.15 & 3.83 & 0.32 & 31.90 & 0.04 & 8 \\
\hline $39 \mathrm{R}-6,80-82$ & 656.20 & & 4.11 & 3.63 & 0.48 & 30.24 & 0.04 & 12 \\
\hline 40R-1, 44-45 & $\begin{array}{l}030.20 \\
657.94\end{array}$ & & $\begin{array}{l}.111 \\
3.26\end{array}$ & 2.20 & $\begin{array}{l}.40 \\
1.06\end{array}$ & $\begin{array}{l}30.24 \\
18.33\end{array}$ & 0.04 & 25 \\
\hline $40 \mathrm{R}-2,128-129$ & $\begin{array}{l}660.28 \\
668\end{array}$ & & 3.53 & 3.22 & 0.31 & $\begin{array}{l}10.53 \\
26.82\end{array}$ & 0.04 & 7 \\
\hline $40 \mathrm{R}-3,113-114$ & 661.63 & & 1.64 & 1.35 & 0.29 & 11.25 & 0.03 & 10 \\
\hline $40 \mathrm{R}-4,118-120$ & 663.18 & & 2.09 & 1.64 & 0.45 & 13.66 & 0.04 & 12 \\
\hline $40 \mathrm{R}-5,71-72$ & 664.21 & & 1.83 & 1.40 & 0.43 & 11.66 & 0.03 & 12 \\
\hline 40R-6, 28-30 & $\begin{array}{l}004.21 \\
665.28\end{array}$ & & $\begin{array}{l}1.83 \\
2.86\end{array}$ & $\begin{array}{l}1.40 \\
2.35\end{array}$ & $\begin{array}{l}0.43 \\
0.51\end{array}$ & $\begin{array}{l}11.00 \\
19.58\end{array}$ & 0.04 & 12 \\
\hline 41R-1,78-79 & $\begin{array}{l}003.20 \\
667.98\end{array}$ & & $\begin{array}{l}.00 \\
3.21\end{array}$ & 2.76 & 0.45 & 22.99 & 0.04 & 12 \\
\hline $41 \mathrm{R}-2,119-120$ & 669.89 & & 1.68 & 1.26 & 0.42 & 10.50 & 0.03 & 12 \\
\hline $41 \mathrm{R}-3,34-35$ & 670.54 & & 1.61 & 1.17 & 0.44 & 9.75 & 0.04 & 12 \\
\hline 41R-4, 108-109 & 672.78 & & 1.39 & 1.04 & 0.35 & 8.66 & 0.03 & 13 \\
\hline $41 \mathrm{R}-5,71-72$ & 673.91 & & 1.22 & 0.83 & 0.39 & 6.91 & 0.03 & 14 \\
\hline 41R-6, 27-28 & $\begin{array}{l}013.91 \\
674.97\end{array}$ & & 1.72 & $\begin{array}{l}0.83 \\
1.30\end{array}$ & 0.42 & $\begin{array}{r}0.91 \\
10.83\end{array}$ & 0.03 & 14 \\
\hline $42 \mathrm{R}-1,28-29$ & 677.08 & & 3.80 & 3.46 & $\begin{array}{l}0.42 \\
0.34\end{array}$ & $\begin{array}{l}10.03 \\
28.82\end{array}$ & 0.03 & 11 \\
\hline $42 \mathrm{R}-2,14-15$ & 678.44 & & 1.78 & 1.48 & 0.30 & 12.33 & 0.03 & 9 \\
\hline $42 \mathrm{R}-3,53-54$ & 680.33 & & 1.10 & 0.77 & 0.33 & 6.41 & 0.03 & 13 \\
\hline $42 \mathrm{R}-4,67-68$ & 681.97 & & 1.30 & 0.86 & 0.44 & 7.16 & 0.03 & 15 \\
\hline $42 \mathrm{R}-5,28-29$ & 683.08 & & 3.80 & 3.36 & 0.44 & 27.99 & 0.04 & 11 \\
\hline $42 \mathrm{R}-6,40-41$ & $\begin{array}{l}083.08 \\
684.70\end{array}$ & & $\begin{array}{l}.80 \\
2.12\end{array}$ & $\begin{array}{l}3.00 \\
1.80\end{array}$ & $\begin{array}{l}0.44 \\
0.32\end{array}$ & 14.99 & $\begin{array}{l}0.04 \\
0.03\end{array}$ & 10 \\
\hline $44 \mathrm{R}-1,131-132$ & 697.41 & & 3.95 & $\begin{array}{l}1.00 \\
3.50\end{array}$ & 0.45 & 29.16 & 0.04 & 11 \\
\hline $44 \mathrm{R}-2,70-71$ & 698.30 & & 3.92 & 3.56 & 0.36 & 29.65 & 0.04 & 10 \\
\hline $44 \mathrm{R}-3,54-55$ & 699.64 & & 2.62 & 2.38 & 0.24 & 19.83 & 0.03 & 9 \\
\hline $44 \mathrm{R}-5,79-81$ & 702.89 & & 2.46 & 2.10 & 0.36 & 17.49 & 0.04 & 10 \\
\hline $47 \mathrm{R}-2,55-57$ & 726.65 & & 2.09 & 1.79 & 0.30 & 14.91 & 0.03 & 10 \\
\hline 51R-1,75-77 & $\begin{array}{l}120.03 \\
763.95\end{array}$ & & 3.05 & 2.71 & 0.34 & $\begin{array}{l}14.91 \\
22.57\end{array}$ & 0.04 & 8 \\
\hline $51 \mathrm{R}-2,49-50$ & 765.19 & & 3.12 & 2.91 & 0.21 & 24.24 & 0.04 & $\begin{array}{l}\circ \\
5\end{array}$ \\
\hline $51 \mathrm{R}-4,140-143$ & 769.10 & & 2.59 & 2.29 & 0.30 & 19.08 & 0.05 & 7 \\
\hline 51R-5, 78-80 & 769.98 & & 1.75 & 0.10 & 1.65 & 0.83 & 0.11 & 16 \\
\hline 51R-6, 77-80 & 771.47 & & 1.22 & 0.12 & 1.10 & 1.00 & 0.08 & 14 \\
\hline 52R-1, 63-64 & 773.53 & & 2.16 & $\begin{array}{l}.12 \\
1.92\end{array}$ & 0.24 & 15.99 & 0.04 & 6 \\
\hline $52 \mathrm{R}-2,24-25$ & 774.64 & & $\begin{array}{l}2.10 \\
2.49\end{array}$ & $\begin{array}{l}1.92 \\
2.20\end{array}$ & $\begin{array}{l}0.24 \\
0.29\end{array}$ & 18.33 & $\begin{array}{l}0.04 \\
0.04\end{array}$ & $\begin{array}{l}0 \\
8\end{array}$ \\
\hline $52 \mathrm{R}-3,63-64$ & $\begin{array}{l}176.04 \\
776.53\end{array}$ & & 3.12 & 2.89 & 0.23 & 24.07 & $\begin{array}{l}0.04 \\
0.04\end{array}$ & $\begin{array}{l}0 \\
5\end{array}$ \\
\hline $52 \mathrm{R}-4,128-129$ & 778.68 & & 4.05 & 3.91 & 0.14 & 32.57 & 0.03 & 5 \\
\hline $52 \mathrm{R}-5,59-60$ & $\begin{array}{l}779.49 \\
-479\end{array}$ & & 3.77 & 3.62 & 0.15 & 30.15 & 0.03 & 6 \\
\hline 53R-1, 19-20 & 782.69 & & 3.17 & 2.98 & 0.19 & 24.82 & 0.05 & 4 \\
\hline 53R-2, 74-75 & 784.74 & & 1.68 & 1.50 & 0.18 & 12.50 & 0.03 & 6 \\
\hline $53 \mathrm{R}-3,53-54$ & 786.03 & & $\begin{array}{l}1.08 \\
2.88\end{array}$ & $\begin{array}{l}1.50 \\
2.77\end{array}$ & $\begin{array}{l}0.18 \\
0.11\end{array}$ & 23.07 & 0.03 & $\begin{array}{l}0 \\
4\end{array}$ \\
\hline 53R-4, $125-126$ & 788.25 & & $\begin{array}{l}.00 \\
1.90\end{array}$ & 1.82 & 0.08 & 15.16 & 0.03 & 3 \\
\hline 53R-5, 100-101 & 789.50 & & 2.29 & $\begin{array}{l}1.02 \\
2.16\end{array}$ & 0.13 & 17.99 & 0.03 & 4 \\
\hline 53R-6, 48-50 & 790.48 & & 2.05 & $\begin{array}{l}.10 \\
1.86\end{array}$ & 0.19 & 15.49 & 0.04 & $\begin{array}{l}4 \\
5\end{array}$ \\
\hline $55 \mathrm{R}-1,31-32$ & 802.11 & & 5.39 & 5.29 & 0.10 & 44.07 & 0.00 & \\
\hline $55 \mathrm{R}-2,21-22$ & 803.51 & & 1.80 & 1.69 & 0.11 & 14.08 & 0.00 & \\
\hline 55R-3, $18-19$ & 804.98 & & 3.26 & 3.12 & 0.14 & 25.99 & 0.00 & \\
\hline
\end{tabular}


Table 1 (continued).

\begin{tabular}{|c|c|c|c|c|c|c|c|c|c|c|c|}
\hline \multirow[b]{2}{*}{$\begin{array}{l}\text { Core, section, } \\
\text { interval }(\mathrm{cm})\end{array}$} & \multirow[b]{2}{*}{$\begin{array}{l}\text { Depth } \\
\text { (mbsf) }\end{array}$} & \multirow[b]{2}{*}{$\begin{array}{l}\text { Age } \\
\text { (Ma) }\end{array}$} & \multirow[b]{2}{*}{$\begin{array}{c}\mathrm{TC} \\
(\mathrm{wt} \%)\end{array}$} & \multirow[b]{2}{*}{$\begin{array}{c}\mathrm{IC} \\
\text { (wt } \%)\end{array}$} & \multirow[b]{2}{*}{$\begin{array}{l}\text { TOC } \\
\text { (wt } \%)\end{array}$} & \multirow[b]{2}{*}{$\begin{array}{l}\mathrm{CaCO}_{3} \\
(\mathrm{wt} \%)\end{array}$} & \multirow[b]{2}{*}{$\begin{array}{c}\mathrm{TN} \\
(\mathrm{wt} \%)\end{array}$} & \multirow[b]{2}{*}{$\begin{array}{c}\mathrm{TOC} / \mathrm{N} \\
\text { ratio }\end{array}$} & \multicolumn{3}{|c|}{ Accumulation rates } \\
\hline & & & & & & & & & $\begin{array}{c}\text { Bulk } \\
\left(\mathrm{g} / \mathrm{cm}^{2} / \mathrm{k} . \mathrm{y} .\right)\end{array}$ & $\begin{array}{c}\text { TOC } \\
\left(\mathrm{g} / \mathrm{cm}^{2} / \mathrm{k} . \mathrm{y} .\right)\end{array}$ & $\begin{array}{c}\text { Carbonate } \\
\left(\mathrm{g} / \mathrm{cm}^{2} / \mathrm{k} . \mathrm{y} .\right)\end{array}$ \\
\hline 55R-4, 65-66 & 806.95 & & 3.98 & 3.86 & 0.12 & 32.15 & 0.02 & 8 & & & \\
\hline $55 \mathrm{R}-5,56-57$ & 808.36 & & 0.70 & 0.63 & 0.07 & 5.25 & 0.00 & & & & \\
\hline 55R-6, 37-38 & 809.67 & & 3.92 & 3.80 & 0.12 & 31.65 & 0.02 & 7 & & & \\
\hline 57R-1, 138-139 & 822.38 & & 4.47 & 4.31 & 0.16 & 35.90 & 0.00 & & & & \\
\hline 57R-2, 137-138 & 823.87 & & 5.57 & 5.42 & 0.15 & 45.15 & 0.00 & & & & \\
\hline $57 \mathrm{R}-3,146-147$ & 825.46 & & 2.83 & 2.79 & 0.04 & 23.24 & 0.00 & & & & \\
\hline $60 \mathrm{R}-1,23-25$ & 850.23 & & 1.89 & 1.89 & 0.00 & 15.74 & 0.00 & & & & \\
\hline $62 \mathrm{R}-1,23-24$ & 869.53 & & 1.51 & 1.36 & 0.15 & 11.33 & 0.00 & & & & \\
\hline $62 \mathrm{R}-2,20-22$ & 871.00 & & 2.85 & 2.75 & 0.10 & 22.91 & 0.00 & & & & \\
\hline $63 \mathrm{R}-1,108-110$ & 879.98 & & 0.87 & 0.89 & 0.00 & 7.41 & 0.00 & & & & \\
\hline $63 \mathrm{R}-2,88-90$ & 881.28 & & 0.87 & 0.74 & 0.13 & 6.16 & 0.00 & & & & \\
\hline $68 \mathrm{R}-1,86-87$ & 925.86 & & 0.64 & 0.61 & 0.03 & 5.08 & 0.00 & & & & \\
\hline 75R-1, 75-76 & 993.05 & & 0.02 & 0.11 & 0.00 & 0.92 & 0.00 & & & & \\
\hline $75 \mathrm{R}-2,130-132$ & 995.10 & & 2.48 & 2.22 & 0.26 & 18.49 & 0.00 & & & & \\
\hline $75 \mathrm{R}-3,59-61$ & 995.89 & & 3.53 & 3.18 & 0.35 & 26.49 & 0.00 & & & & \\
\hline $75 \mathrm{R}-4,31-33$ & 997.11 & & 2.39 & 2.20 & 0.19 & 18.33 & 0.00 & & & & \\
\hline $76 \mathrm{R}-1,108-110$ & 1003.08 & & 1.89 & 1.64 & 0.25 & 13.66 & 0.00 & & & & \\
\hline 77R-1, 54-55 & 1012.14 & & 0.03 & 0.13 & 0.00 & 1.08 & 0.00 & & & & \\
\hline $78 \mathrm{R}-1,50-51$ & 1021.70 & & 2.78 & 2.50 & 0.28 & 20.83 & 0.00 & & & & \\
\hline $79 \mathrm{R}-1,40-41$ & 1031.30 & & 0.23 & 0.31 & 0.00 & 2.58 & 0.00 & & & & \\
\hline $80 \mathrm{R}-1,17-18$ & 1040.77 & & 1.78 & 1.64 & 0.14 & 13.66 & 0.00 & & & & \\
\hline $83 R-1,56-57$ & 1070.16 & & 0.21 & 0.48 & 0.00 & 4.00 & 0.00 & & & & \\
\hline $84 \mathrm{R}-1,40-41$ & 1079.70 & & 0.35 & 0.44 & 0.00 & 3.67 & 0.00 & & & & \\
\hline 88R-1, 47-49 & 1118.27 & & 2.62 & 2.51 & 0.11 & 20.91 & 0.00 & & & & \\
\hline 89R-2, 135-137 & 1130.25 & & 4.82 & 4.88 & 0.00 & 40.65 & 0.00 & & & & \\
\hline $89 \mathrm{R}-3,11-12$ & 1130.51 & & 4.83 & 4.42 & 0.41 & 36.82 & 0.00 & & & & \\
\hline $89 \mathrm{R}-3,54-56$ & 1130.94 & & 3.70 & 3.43 & 0.27 & 28.57 & 0.00 & & & & \\
\hline $89 R-4,50-51$ & 1132.40 & & 4.60 & 4.33 & 0.27 & 36.07 & 0.00 & & & & \\
\hline $90 \mathrm{R}-2,25-26$ & 1138.85 & & 3.12 & 2.23 & 0.89 & 18.58 & 0.03 & 33 & & & \\
\hline $90 \mathrm{R}-3,24-25$ & 1140.34 & & 2.89 & 1.79 & 1.10 & 14.91 & 0.04 & 26 & & & \\
\hline $91 \mathrm{R}-1,26-27$ & 1146.76 & & 2.21 & 1.34 & 0.87 & 11.16 & 0.04 & 23 & & & \\
\hline 92R-1, 97-98 & 1157.17 & & 1.91 & 1.49 & 0.42 & 12.41 & 0.02 & 22 & & & \\
\hline $93 \mathrm{R}-1,131-132$ & 1167.11 & & 0.51 & 0.37 & 0.14 & 3.08 & 0.02 & 8 & & & \\
\hline 93R-2, 20-21 & 1167.50 & & 0.80 & 0.63 & 0.17 & 5.25 & 0.01 & 17 & & & \\
\hline
\end{tabular}

Notes: $\mathrm{TC}=$ total carbon, $\mathrm{IC}=$ inorganic carbon, $\mathrm{TOC}=$ total organic carbon, and $\mathrm{TN}=$ total nitrogen.

The composition and amount of organic matter in the sediments of Site 918 very well mirror the long-term changes in climatic development and paleoenvironment of the southeast Greenland region. Paleoceanographic conditions are strongly dependent on the intensity of the East Greenland Current (Larsen, Saunders, Clift, et al., 1994) and, thus, influence the accumulation and preservation of organic carbon.

During Eocene and Oligocene times (lithologic Unit III, 1180$800 \mathrm{mbsf}$ ), the siliciclastic as well as organic sediment parameters (see "Results" section above) indicate relatively warm climatic conditions and shallow water depth $(<1500 \mathrm{~m})$. This led to the deposition of mainly terrigenous organic material derived from the surrounding coastal areas. Dense vegetation resulted in enhanced deposition of plant material confirmed by vitrinites and long-chain $n$-alkanes (Fig. 2; Table 3). Finely dispersed wood pieces also indicate transport by rivers or occasional driftwood. Marine production was probably very low during this time. No evidence for increased productivity as diatom or nannofossil remains could be found in the sediment parameters. Additionally, low sedimentation rates (Larsen, Saunders, Clift, et al., 1994) and oxic water column conditions may have caused an intense degradation of the labile marine organic matter (cf. Stein, 1991).

In the lower to upper Miocene sediments of lithologic Unit II ( 800-600 mbsf), increased organic carbon contents and amounts of planktonic organisms point to an enhanced surface-water productivity. This is supported by microscopic findings (strong background fluorescence and a lack of terrigenous macerals). The alkane distribution, hydrogen index values, and $\mathrm{C} / \mathrm{N}$ ratios, however, indicate a mixture of marine and terrigenous organic material (Figs. 2, 3). During this time interval, a highly oxidizing water column (bioturbation) and extremely low sedimentation rates support the diagenesis and remineralization of the organic material. The depositional conditions are comparable to a hemipelagic oxic environment, which is dominated by marine organic matter with a minor terrigenous organic input.
A dramatic change in depositional conditions occurred at Site 918 at about $5 \mathrm{Ma}$ ( 500 mbsf; Larsen, Saunders, Clift, et al., 1994). The sedimentation rate of siliciclastic material increased to values as much as $19.5 \mathrm{~cm} / \mathrm{k} . \mathrm{y}$., and biogenic carbonate production almost terminated. The flux of organic carbon increased (mean values of about $0.7 \mathrm{~g} / \mathrm{cm}^{2} / \mathrm{k}$.y.; Fig. 4), and the preservation of organic particles is moderate. Well-preserved alginites are sparse. Climatic conditions probably turned to colder temperatures (increase in dropstone occurrence), and the dense vegetation cover disappeared. Thus, terrigenous organic material is only a minor component of the organic sediment fraction. This is supported by the $n$-alkane pattern, hydrogen index values, and microscopic observations (Figs. 2, 3; Table 3). Rapid burial of the organic matter may have led to a better preservation and higher flux rates during this time interval.

\section{ACKNOWLEDGMENTS}

Helpful data discussions with R. Stein, K. Fahl, and C. Schubert are gratefully acknowledged. This study was financially supported by the Deutsche Forschungs-gemeinschaft (grant no. STE 412/7).

\section{REFERENCES}

Eldholm, O., Thiede, J., Taylor, E., et al., 1987. Proc. ODP, Init. Repts., 104: College Station, TX (Ocean Drilling Program).

Espitalié, J., Laporte, J.L., Leplat, P., Madec, M., Marquis, F., Paulet, J., and Boutefeu, A., 1977. Méthode rapide de caractérisation des roches mères, de leur potentiel pétrolier et de leur degré d'évolution. Rev. Inst. Fr. Pet., 32:23-42.

Farrimond, P., Poynter, J.G., and Eglinton, G., 1990. Molecular composition of sedimentary lipids off the Peru margin, Leg 112. In Suess, E., von Huene, R., et al., Proc. ODP, Sci. Results, 112: College Station, TX (Ocean Drilling Program), 539-546.

Froelich, P.N., 1980. Analysis of organic carbon in marine sediments. Limnol. Oceanogr., 25:564-572. 
Hutton, A.C., Kantsler, A.J., Cook, A.C., and Kirdy, D.M., 1980. Organic matter in oil shales. APEA J., 20:44-67.

Katz, B.J., 1983. Limitations of "Rock-Eval" pyrolysis for typing organic matter. Org. Geochem., 4:195-199.

Larsen, H.C., Saunders, A.D., Clift, P.D., et al., 1994. Proc. ODP, Init. Repts., 152: College Station, TX (Ocean Drilling Program).

Peters, K.E., 1986. Guidelines for evaluating petroleum source rock using programmed pyrolysis. AAPG Bull., 70:318-329.

Prahl, F.G., and Muehlhausen, L.A., 1989. Lipid biomarkers as geochemical tools for paleoceanographic study. In Berger, W.H., Smetacek, V.S., and Wefer, G. (Eds.), Productivity of the Ocean: Present and Past. Dahlem Workshop, Life Sci. Res. Rep., 44:271-289.

Scheffer, F., and Schachtschabel, P., 1984. Lehrbuch der Bodenkunde: Stuttgart (Enke).

Srivastava, S.P., Arthur, M., Clement, B., et al., 1987. Proc. ODP, Init. Repts., 105: College Station, TX (Ocean Drilling Program).

Stach, E., Mackowsky, M.-T., Teichmüller, M., Taylor, G.H., Chandra, D., and Teichmüller, R., 1982. Stach's Textbook of Coal Petrology (3rd ed.): Berlin (Gebrüder Borntraeger).

Stax, R., 1994. Zyklische Sedimentaation von organischem Kohlenstoff in der Japan See: Anzeiger für Änderungen von Pläoozeanographie und Paläoklima im Spätkänozoikum. Ber. Univ. Bremen, Univ. Druckerei, 47.
Stein, R., 1991. Accumulation of Organic Carbon in Marine Sediments: Results from the Deep Sea Drilling Project/Ocean Drilling Program $(D S D P / O D P)$. Lect. Notes Earth Sci., 34: Heidelberg (Springer-Verlag).

Tissot, B.P., and Welte, D.H., 1984. Petroleum Formation and Occurrence (2nd ed.): Heidelberg (Springer-Verlag).

van Andel, T.H., Heath, G.R., and Moore, T.C., Jr., 1975. Cenozoic history and paleoceanography of the central equatorial Pacific Ocean: a regional synthesis of Deep Sea Drilling Project data. Mem.-Geol. Soc. Am., 143.

Weliky, K., Suess, E., Ungerer, C.A., Müller, P.J., and Fischer, K., 1983. Problems with accurate carbon measurements in marine sediments and particulate matter in seawater: a new approach. Limnol. Oceanogr., $28: 1252-1259$.

Date of initial receipt: 9 November 1995

Date of acceptance: 29 May 1996

Ms 152SR-226

Table 2. Results of the Rock-Eval pyrolysis.

\begin{tabular}{|c|c|c|c|c|c|c|c|c|}
\hline $\begin{array}{l}\text { Core, section, } \\
\text { interval }(\mathrm{cm})\end{array}$ & $\begin{array}{l}\text { Depth } \\
\text { (mbsf) }\end{array}$ & $\begin{array}{l}\text { TOC } \\
(w t \%)\end{array}$ & $\mathrm{S}_{1}$ & $\mathrm{~S}_{2}$ & $\mathrm{~S}_{3}$ & HI & OI & $\begin{array}{l}\mathrm{T}_{\max } \\
\left({ }^{\circ} \mathrm{C}\right)\end{array}$ \\
\hline \multicolumn{9}{|l|}{ 152-918A- } \\
\hline $3 \mathrm{H}-3,30-31$ & 14.60 & 0.53 & 0.06 & 0.59 & 0.47 & 111 & 89 & 515 \\
\hline $4 \mathrm{H}-1,134-136$ & 22.14 & 0.47 & 0.09 & 0.39 & 0.73 & 82 & 156 & 432 \\
\hline $5 \mathrm{H}-3,11-13$ & 33.41 & 0.53 & 0.12 & 0.32 & 1.14 & 61 & 216 & 422 \\
\hline $6 \mathrm{H}-4,58-60$ & 44.88 & 0.42 & 0.32 & 0.39 & 1.08 & 92 & 258 & 441 \\
\hline $8 \mathrm{H}-3,120-122$ & 63.00 & 0.38 & 0.09 & 0.33 & 0.09 & 88 & 24 & 451 \\
\hline $11 \mathrm{H}-1,141-143$ & 79.21 & 0.40 & 0.34 & 0.47 & 0.21 & 118 & 53 & 433 \\
\hline $11 \mathrm{H}-3,21-23$ & 90.51 & 0.51 & 0.22 & 0.53 & 0.67 & 104 & 132 & 412 \\
\hline $13 \mathrm{H}-2,33-35$ & 108.13 & 0.44 & 0.13 & 0.42 & 1.17 & 95 & 265 & 432 \\
\hline $15 \mathrm{H}-3,14-16$ & 128.44 & 0.37 & 0.90 & 0.29 & 0.83 & 79 & 223 & 432 \\
\hline $17 \mathrm{H}-6,6-8$ & 150.36 & 0.42 & 0.32 & 0.20 & 1.19 & 48 & 284 & 500 \\
\hline $17 \mathrm{H}-6,122-123$ & 151.52 & 0.68 & 0.05 & 0.51 & 1.08 & 75 & 160 & 438 \\
\hline $19 \mathrm{H}-1,119-121$ & 162.99 & 0.29 & 0.08 & 0.16 & 0.23 & 56 & 79 & 436 \\
\hline $21 \mathrm{X}-1,126-128$ & 182.76 & 0.39 & 0.32 & 0.26 & 0.62 & 66 & 160 & 435 \\
\hline $23 \mathrm{X}-6,103-105$ & 208.33 & 0.40 & 0.42 & 0.38 & 0.22 & 95 & 54 & 423 \\
\hline $25 \mathrm{X}-1,107-108$ & 218.67 & 0.65 & 0.04 & 0.23 & 1.09 & 36 & 169 & 517 \\
\hline $25 \mathrm{X}-2,21-23$ & 219.31 & 0.43 & 0.32 & 0.52 & 0.57 & 121 & 132 & 432 \\
\hline $25 \mathrm{X}-4,107-108$ & 223.17 & 1.25 & 0.17 & 1.01 & 2.96 & 81 & 238 & 430 \\
\hline $26 \mathrm{X}-1,21-22$ & 226.71 & 1.30 & 0.07 & 1.07 & 3.72 & 83 & 287 & 424 \\
\hline $27 X-4,130-132$ & 241.49 & 0.52 & 0.12 & 0.50 & 1.18 & 96 & 226 & 452 \\
\hline $31 X-2,34-36$ & 272.44 & 0.38 & 0.33 & 0.13 & 0.21 & 35 & 56 & 418 \\
\hline $37 X-2,73-75$ & 317.33 & 0.51 & 0.09 & 0.38 & 0.41 & 75 & 81 & 398 \\
\hline $37 \mathrm{X}-2,124-125$ & 317.84 & 0.50 & 0.07 & 0.11 & 2.54 & 22 & 512 & 395 \\
\hline \multicolumn{9}{|l|}{ 152-918D- } \\
\hline 11R-1, 101-102 & 387.11 & 0.53 & 0.05 & 0.29 & 0.29 & 55 & 55 & 442 \\
\hline $18 \mathrm{R}-1,45-47$ & 448.65 & 0.41 & 0.04 & 0.36 & 0.59 & 87 & 145 & 421 \\
\hline $25 \mathrm{R}-1,83-85$ & 513.63 & 0.44 & 0.21 & 0.27 & 0.73 & 62 & 166 & 436 \\
\hline $27 \mathrm{R}-2,140-142$ & 535.00 & 0.39 & 0.09 & 0.20 & 0.90 & 51 & 232 & 422 \\
\hline $37 \mathrm{R}-2,97-99$ & 631.07 & 0.51 & 0.32 & 0.17 & 0.48 & 34 & 95 & 443 \\
\hline $38 \mathrm{R}-4,105-106$ & 643.75 & 1.39 & 0.12 & 0.07 & 1.45 & 5 & 105 & 417 \\
\hline $40 \mathrm{R}-1,44-45$ & 657.94 & 1.06 & 0.08 & 0.22 & 1.69 & 21 & 160 & 413 \\
\hline $40 \mathrm{R}-4,5-7$ & 662.05 & 0.33 & 0.12 & 0.18 & 0.28 & 55 & 85 & 421 \\
\hline $40 \mathrm{R}-6,28-30$ & 665.28 & 0.51 & 0.09 & 0.16 & 1.40 & 31 & 274 & 414 \\
\hline $42 \mathrm{R}-5,138-140$ & 684.18 & 0.51 & 0.52 & 0.23 & 0.67 & 45 & 132 & 432 \\
\hline $51 \mathrm{R}-5,78-80$ & 769.98 & 1.65 & 0.26 & 1.08 & 1.41 & 65 & 85 & 409 \\
\hline $51 \mathrm{R}-6,77-80$ & 771.47 & 1.10 & 0.32 & 0.54 & 0.87 & 49 & 79 & 422 \\
\hline $53 \mathrm{R}-1,108-110$ & 783.58 & 0.38 & 0.20 & 0.14 & 0.86 & 38 & 225 & 436 \\
\hline $55 \mathrm{R}-2,106-108$ & 804.36 & 0.35 & 0.09 & 0.23 & 0.43 & 65 & 123 & 455 \\
\hline $57 \mathrm{R}-3,26-28$ & 824.26 & 0.32 & 0.07 & 0.17 & 0.71 & 54 & 221 & 419 \\
\hline 75R-1, 7-9 & 992.37 & 0.36 & 0.21 & 0.12 & 0.94 & 32 & 261 & 435 \\
\hline 83R-1, 96-98 & 1070.56 & 0.29 & 0.90 & 0.16 & 0.25 & 55 & 85 & 422 \\
\hline $90 \mathrm{R}-2,25-26$ & 1138.85 & 0.89 & 0.09 & 0.49 & 1.45 & 55 & 163 & 435 \\
\hline $90 \mathrm{R}-3,24-25$ & 1140.34 & 1.10 & 0.09 & 0.59 & 1.50 & 54 & 137 & 430 \\
\hline $91 \mathrm{R}-1,26-27$ & 1146.76 & 0.87 & 0.58 & 0.50 & 1.40 & 58 & 162 & 432 \\
\hline 95R-1, 29-31 & 1180.69 & 0.51 & 0.12 & 0.21 & 0.29 & 41 & 56 & 432 \\
\hline
\end{tabular}

Notes: $\mathrm{S}_{1}$ (mg HC/g sediment), $\mathrm{S}_{2}\left(\mathrm{mg} \mathrm{HC} / \mathrm{g}\right.$ sediment), and $\mathrm{S}_{3}\left(\mathrm{mg} \mathrm{CO}_{2} / \mathrm{g}\right.$ sediment$) . \mathrm{HI}=$ hydrogen index $(\mathrm{mg} \mathrm{HC} / \mathrm{g} \mathrm{TOC})$, and OI $=$ oxygen index $(\mathrm{mg} \mathrm{CO} / \mathrm{g} \mathrm{TOC})$ 
Table 3. Microscopic results of selected resin impregnated specimens.

\begin{tabular}{|c|c|c|c|c|c|c|c|c|c|c|c|}
\hline $\begin{array}{l}\text { Core, section, } \\
\text { interval }(\mathrm{cm})\end{array}$ & $\begin{array}{l}\text { Depth } \\
\text { (mbsf) }\end{array}$ & $\begin{array}{c}\text { TOC } \\
\text { (wt } \%)\end{array}$ & $\begin{array}{c}\mathrm{HI} \\
\text { (mg HC/g C) }\end{array}$ & $\begin{array}{c}\mathrm{OI} \\
(\mathrm{mg} \mathrm{CO} / \mathrm{g} \mathrm{C})\end{array}$ & $\mathrm{T}_{\max }\left({ }^{\circ} \mathrm{C}\right)$ & Alginite & Liptodetrinite & Vitrinite & Vitrodetrinite & Pollenite & $\begin{array}{l}\text { Background } \\
\text { fluorescence }\end{array}$ \\
\hline \multicolumn{12}{|l|}{ 152-918A- } \\
\hline $17 \mathrm{H}-6,6-8$ & 150.36 & 0.42 & 48 & 284 & 500 & $\mathrm{X}$ & $\mathrm{X}$ & $\mathrm{O}$ & $\mathrm{X}$ & $\mathrm{O}$ & $\mathrm{X}$ \\
\hline $23 \mathrm{X}-6,103-105$ & 208.33 & 0.40 & 95 & 54 & 423 & $\mathrm{O}$ & $\mathrm{X}$ & $\mathrm{O}$ & $\mathrm{O}$ & $\mathrm{O}$ & $\mathrm{X}$ \\
\hline $31 X-2,34-36$ & 272.44 & 0.38 & 35 & 56 & 418 & $\mathrm{O}$ & $\mathrm{X}$ & $\mathrm{O}$ & $\mathrm{O}$ & $\mathrm{X}$ & $\mathrm{XX}$ \\
\hline \multicolumn{12}{|l|}{ 152-918D- } \\
\hline $18 \mathrm{R}-1,45-47$ & 448.65 & 0.41 & 87 & 145 & 421 & $\mathrm{X}$ & $\mathrm{X}$ & $\mathrm{O}$ & $\mathrm{O}$ & $\mathrm{O}$ & $\mathrm{X}$ \\
\hline $27 \mathrm{R}-2,140-142$ & 535.00 & 0.39 & 51 & 232 & 422 & $\mathrm{O}$ & $X X$ & $\mathrm{O}$ & $\mathrm{O}$ & $\mathrm{X}$ & $\mathrm{X}$ \\
\hline $37 \mathrm{R}-2,97-99$ & 631.07 & 0.51 & 34 & 95 & 443 & $\mathrm{XX}$ & $\mathrm{XX}$ & $\mathrm{X}$ & $\mathrm{O}$ & $\mathrm{O}$ & $\mathrm{XX}$ \\
\hline $42 \mathrm{R}-5,138-140$ & 684.18 & 0.51 & 45 & 132 & 432 & $\mathrm{XX}$ & $\mathrm{X}$ & $\mathrm{X}$ & $\mathrm{X}$ & $\mathrm{O}$ & $\mathrm{XX}$ \\
\hline $55 \mathrm{R}-2,106-108$ & 804.36 & 0.35 & 65 & 123 & 455 & $\mathrm{X}$ & $\mathrm{O}$ & $\mathrm{X}$ & $\mathrm{XX}$ & $\mathrm{O}$ & $\mathrm{X}$ \\
\hline $75 \mathrm{R}-1,7-9$ & 992.37 & 0.36 & 32 & 261 & 435 & $\mathrm{O}$ & $\mathrm{O}$ & $\mathrm{X}$ & $\mathrm{X}$ & $\mathrm{X}$ & $\mathrm{O}$ \\
\hline $83 R-1,96-98$ & 1070.56 & 0.29 & 55 & 85 & 422 & $\mathrm{X}$ & $\mathrm{X}$ & $\mathrm{XX}$ & $\mathrm{XX}$ & $\mathrm{X}$ & $\mathrm{O}$ \\
\hline $95 \mathrm{R}-1,29-31$ & 1180.69 & 0.51 & 41 & 56 & 432 & $\mathrm{O}$ & $\mathrm{O}$ & $\mathrm{X}$ & $\mathrm{XX}$ & $\mathrm{X}$ & $\mathrm{O}$ \\
\hline
\end{tabular}

Notes: $\mathrm{XX}=$ abundant, $\mathrm{X}=$ moderate to minor, and $\mathrm{O}=$ minor to absent.
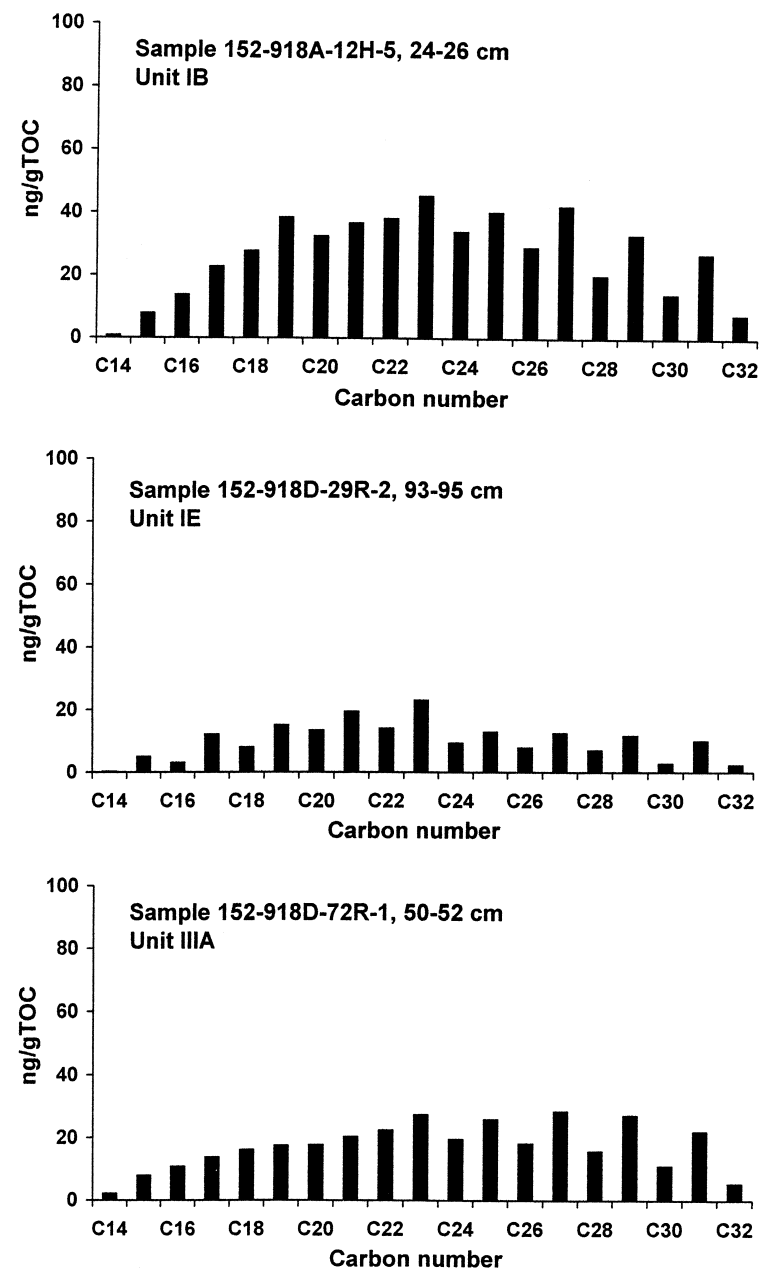
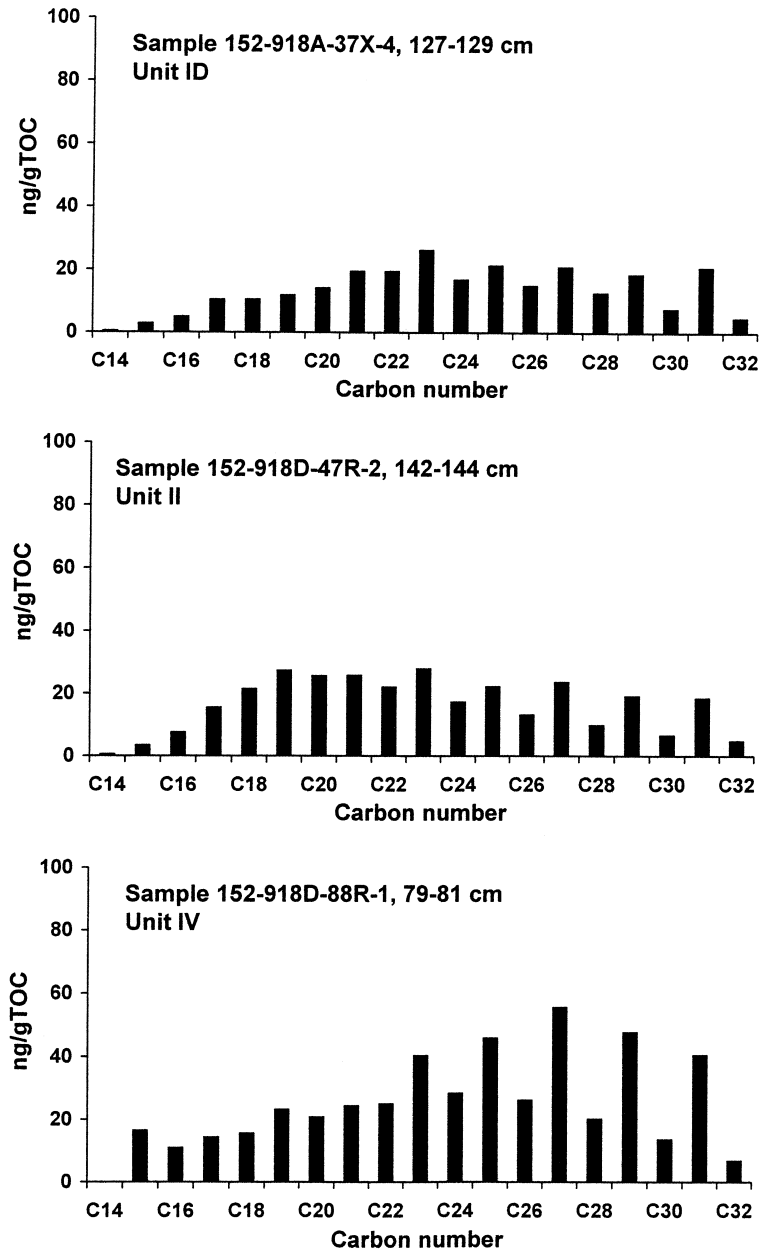

Figure 3. $N$-alkane data listed for six samples from different lithologic units (Larsen, Saunders, Clift, et al., 1994). 
Table 4. Distribution of $\boldsymbol{n}$-alkanes in samples from Site 918.

\begin{tabular}{|c|c|c|c|c|c|c|}
\hline $\begin{array}{l}\text { Hole: } \\
\text { Core, section: } \\
\text { Interval }(\mathrm{cm}) \text { : }\end{array}$ & $\begin{array}{c}918 \mathrm{~A} \\
12 \mathrm{H}-5 \\
24 \\
\text { (ng/g TOC) }\end{array}$ & $\begin{array}{c}918 \mathrm{~A} \\
37 \mathrm{X}-4 \\
73 \\
\text { (ng/g TOC) }\end{array}$ & $\begin{array}{c}918 \mathrm{~A} \\
29 \mathrm{R}-2 \\
93 \\
\text { (ng/g TOC) }\end{array}$ & $\begin{array}{c}918 \mathrm{~A} \\
47 \mathrm{R}-2 \\
142 \\
\text { (ng/g TOC) }\end{array}$ & $\begin{array}{c}918 \mathrm{~A} \\
72 \mathrm{R}-1 \\
50 \\
\text { (ng/g TOC) }\end{array}$ & $\begin{array}{c}918 \mathrm{~A} \\
88 \mathrm{R}-1 \\
79 \\
\text { (ng/g TOC) }\end{array}$ \\
\hline $\begin{array}{l}\mathrm{C}_{14} \\
\mathrm{C}_{15} \\
\mathrm{C}_{16} \\
\mathrm{C}_{17} \\
\mathrm{C}_{18} \\
\mathrm{C}_{19} \\
\mathrm{C}_{20} \\
\mathrm{C}_{21} \\
\mathrm{C}_{22} \\
\mathrm{C}_{23} \\
\mathrm{C}_{24} \\
\mathrm{C}_{25} \\
\mathrm{C}_{26} \\
\mathrm{C}_{27} \\
\mathrm{C}_{28} \\
\mathrm{C}_{29} \\
\mathrm{C}_{30} \\
\mathrm{C}_{31} \\
\mathrm{C}_{32}\end{array}$ & $\begin{array}{r}0.75 \\
7.83 \\
13.70 \\
22.64 \\
27.64 \\
38.38 \\
32.42 \\
36.57 \\
38.06 \\
45.19 \\
33.91 \\
40.08 \\
28.91 \\
42.11 \\
19.98 \\
32.96 \\
14.13 \\
26.89 \\
7.53\end{array}$ & $\begin{array}{r}0.20 \\
5.00 \\
3.02 \\
12.09 \\
8.00 \\
15.02 \\
13.33 \\
19.32 \\
13.98 \\
23.00 \\
9.35 \\
12.85 \\
7.77 \\
12.43 \\
6.95 \\
11.60 \\
2.78 \\
9.93 \\
2.36\end{array}$ & $\begin{array}{r}0.44 \\
2.82 \\
4.93 \\
10.39 \\
10.48 \\
11.82 \\
14.14 \\
19.41 \\
19.41 \\
26.19 \\
16.82 \\
21.28 \\
14.85 \\
20.84 \\
12.62 \\
18.52 \\
7.53 \\
20.66 \\
4.67\end{array}$ & $\begin{array}{r}0.54 \\
3.36 \\
7.52 \\
15.44 \\
21.37 \\
27.30 \\
25.57 \\
25.69 \\
21.99 \\
27.79 \\
17.17 \\
22.11 \\
13.10 \\
23.47 \\
9.79 \\
19.02 \\
6.54 \\
18.41 \\
4.78\end{array}$ & $\begin{array}{r}2.19 \\
7.93 \\
10.76 \\
13.73 \\
16.18 \\
17.47 \\
17.70 \\
20.30 \\
22.44 \\
27.40 \\
19.54 \\
25.87 \\
18.16 \\
28.39 \\
15.72 \\
27.17 \\
10.99 \\
21.98 \\
5.39\end{array}$ & $\begin{array}{r}16.47 \\
10.94 \\
14.32 \\
15.59 \\
23.18 \\
20.78 \\
24.32 \\
24.95 \\
40.27 \\
28.37 \\
45.84 \\
26.09 \\
55.46 \\
20.14 \\
47.61 \\
13.56 \\
40.40 \\
6.79\end{array}$ \\
\hline
\end{tabular}

Note: $\mathrm{C}_{14}$ through $\mathrm{C}_{32}=$ carbon numbers.

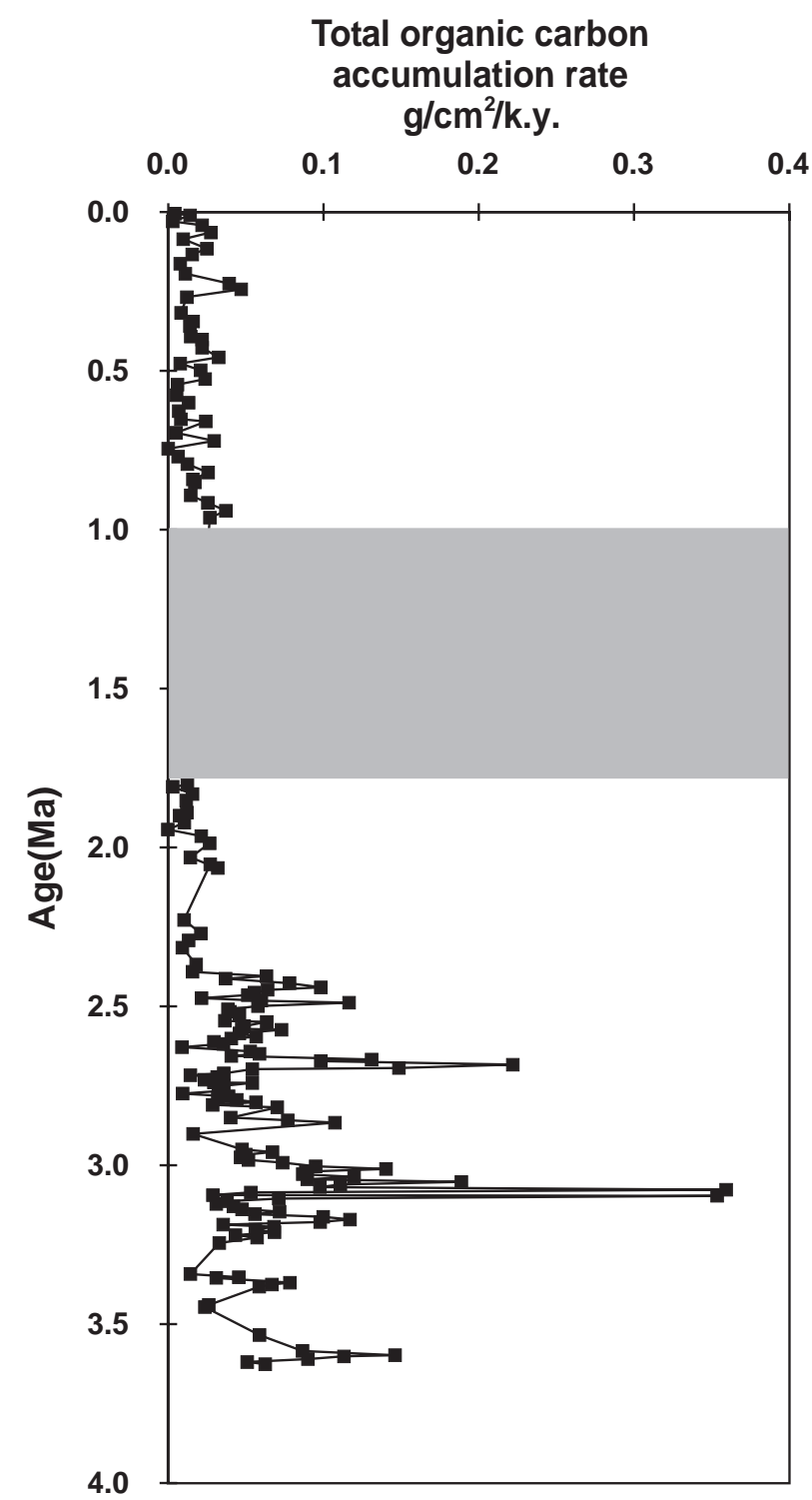

Figure 4. Mass accumulation rates of total organic carbon vs. age of Site 918.

Shaded area indicates a possible hiatus (Larsen, Saunders, Clift, et al., 1994). 